\title{
Equitable and effective climate policy: Integrating less developed countries into a global climate agreement
}

\author{
Lucas Bretschger $^{1}$ - Alexandra Vinogradova ${ }^{2}$
}

Published online: 2 August 2015

(C) Springer-Verlag Berlin Heidelberg 2015

\begin{abstract}
The paper derives general rules for equitable burden sharing in international climate policy. The focus is on a new social climate contract between developed and less developed countries (LDCs) which preserves competitiveness of the former and the "right to development" of the latter. We formally derive conditions under which an LDC keeps the "right to development" but voluntarily agrees to participate in stringent international climate policy. Two types of policies are analyzed, one with a predefined transfer and the other with a transfer that is tied to emissionscontrol efforts. We show that offering only one or the other option is inefficient. Chances for a comprehensive agreement are higher when a menu of policy options is available. The number and diversity of LDCs willing to join a global climate treaty is higher when a variety of policy alternatives is available.
\end{abstract}

Keywords Climate policy · Less developed countries · Equitable burden sharing · Right to development · International climate agreement

Valuable comments by Thomas Gries and Paul Welfens are gratefully acknowledged.

Lucas Bretschger

lbretschger@ethz.ch

Alexandra Vinogradova

avinogradova@ethz.ch

1 CER-ETH Center of Economic Research at ETH Zurich,

ZUE F7: Zürichbergstrasse 18, CH-8092 Zurich, Switzerland

2 CER-ETH Center of Economic Research at ETH Zurich,

ZUE F13: Zürichbergstrasse 18, CH-8092 Zurich, Switzerland 


\section{Introduction}

By ratifying the United Nations Framework Convention on Climate Change, the world community, represented by 196 parties, committed to establish binding international rules for greenhouse gas emissions. For over two decades, international climate policy has aimed at reaching an agreement on emission reduction targets. According to the general expectations, a climate treaty should be efficient, fair, and global. The Kyoto protocol, adopted in Japan in 1997, proved that such an agreement is feasible in principle. But, in fact, the protocol never fulfilled its main purpose of implementing a comprehensive climate policy. It only covers a part, and even a sharply decreasing part, of world emissions. The agreement places a heavy burden on developed nations, strictly limiting the number of countries taking responsibility for global warming. This was done in recognition of historic development. Indeed, high greenhouse gas concentration in the atmosphere is a result of more than 150 years of industrial activity, which was mostly undertaken in the wealthy nations. However, in the last years, economic development has been very dynamic and carbon intensive. In particular, emerging economies have increased their emissions drastically. Moreover, some countries never ratified the Kyoto protocol and some parties pulled out of the process in the meantime. Hence, a new agreement for effective global climate policy has to be designed and decided. It has to build on a much broader approach than its predecessor, providing a truly global framework.

When integrating the developed and the less developed part of the world economy in a single policy framework, two basic issues have to be addressed. First, we have to address the concern of threatened competitiveness, arising when some countries have to adjust their carbon prices due to a climate agreement while other countries do not participate in the global climate policy. On several occasions, European policymakers expressed their readiness to apply trade restrictions on countries which aim at free riding on international climate policies. For instance, Manuel Barroso in his interview to The Times said: "We do not want to put our energy-intensive industries in a situation of disadvantage in competition terms, that is why we will have measures that we are ready to take if there is not a global climate agreement" (March 2008). Former French president Nicolas Sarkozy said that EU must examine the possibility of "taxing products imported from countries that do not comply with the Kyoto protocol. We have imposed environmental standards on our producers. It is not normal that their competitors should be completely exempted...Environmental dumping is not fair" (October 2007). In particular, the so-called "border-adjustment measures" were a hot discussion topic and were viewed as indispensable for a climate legislation to pass in the US Congress. Hence, to provide an efficient solution to global warming, all the countries, including the emerging economies and the less developed countries (LDCs), have to be included in a global agreement.

The second main issue with the global climate policy is that all the parties, but especially the less developed countries, need to be treated in an equitable and fair manner. In 1986, the General Assembly of the United Nations decided to adopt the Declaration on the Right to Development, which includes various central development aspects ranging from individual and political freedom to economic progress. Hence, climate policy should not prevent LDCs from developing their economies and 
living standards. Global climate mitigation itself is also crucial for development of the LDCs, which are more vulnerable to climate change due to geography and the lack of capital and knowledge for adaptation.

For international climate policy, the year 2015 is very important, if not decisive. The recently adopted "Lima call for climate action" underscores the commitment "to reaching an ambitious agreement in 2015 that reflects the principle of common but differentiated responsibilities and respective capabilities." At the next conference of the parties a new agreement should finally be reached. This policy shift is broadly expected, while many other policy changes with large global effects have often come unexpectedly. Indeed, the fall of the Berlin wall, the deep economic reforms in China, and the collapse of the Bretton-Woods system were largely unanticipated, at least in terms of timing. But in all cases, general conditions for new policy rules were prepared, time was ripe for a change. In climate policy, conditions have been prepared for a long time, but a major breakthrough at the end of the year is still not certain. The negotiating parties are very diverse and so are their economic interests. In principal, the concept of "differentiated responsibilities" adopted with the United Nations Framework Convention on Climate Change (UNFCCC) should take this into full consideration. Accordingly, the "Lima call for climate action" urges countries to explain "how the party considers that its intended nationally determined contribution is fair and ambitious, in light of its national circumstances, and how it contributes towards achieving the objective of the convention ..." It is thus decisive to achieve the integration of all the countries by respecting their specific conditions and capacities to contribute to international climate policy. Moreover, it is crucial to support LDCs in their effort for climate mitigation. In May 2015, French President Francois Hollande said that there will be no global climate change deal "without finance for poor nations" and that "ramping up climate finance for developing countries to $\$ 100$ billion a year by 2020 will be essential." He added that "developing countries won't accept an agreement if they do not get any financial support .." Hence, success at the upcoming climate change summit should definitely include a significant finance package to the less and least developed countries. Moreover, financial aid can be used when developed countries wish to trade part of their climate policy obligations with the LDCs.

The present paper develops broad and generally applicable concepts for effective and equitable climate policy. We proceed in two steps. In a first short part we analyze rules for global and equitable burden sharing, using the global carbon budget approach and basic equity principles. We analyze the rules to derive fair contributions of developed, emerging, and less developed economies. From this analysis it emerges that LDCs deserve special attention and treatment. Indeed, specific incentives in terms of transfers and assistance are warranted to preserve the LDCs right to development when joining a climate agreement. To support the argument, the analytical part of the paper focuses on economic development in LDCs. Specifically, we highlight the conditions under which an LDC voluntarily agrees to join the international climate policy, while keeping what has generally been called the "right to development." This important term, often loosely interpreted in applied and policy work, will be given a precise economic meaning in our framework. The purpose of the model is to establish the minimum conditions for voluntary compliance and to 
analyze the LDC's optimal response to any changes in the conditions it faces. We analyze two types of support offers to LDCs: One where a predefined transfer is initiated on the date of compliance with a predetermined emissions target; and the other where the amount transferred is tied to emissions-control efforts. The main result of the model is that offering one or the other option is inefficient. We show that the chances of an LDC voluntarily complying with global environmental standards are higher when a menu of policy options is on the table. The direct implication of this result is that the number and diversity of countries willing to join a global climate treaty is also higher when a variety of alternatives is available instead of just one policy offer.

The remainder of the paper is organized as follows. Section 2 analyzes equitable ways for the integration of all the countries in a global climate agreement. Section 3 sets up a macroeconomic model for development of an LDC; two types of policies in terms of offers to LDCs are introduced. Section 4 studies the conditions under which LDCs voluntarily join a climate agreement. Section 5 is devoted to more detailed policy analysis. Section 6 concludes.

\section{Global burden sharing}

Deriving efficient climate policies requires consideration of a very long time horizon. This is because greenhouse gas emissions cause economic damages only after a major time lag. On the contrary, political decision making is mostly directed at shorter time horizons, favorably reacting on events which are evident, pressing, and raising general concerns in the electorate. Grave uncertainties about climate shocks in the future further complicate matters in the case of climate policy; both the arrival and the size of the shocks seem to be unpredictable. While theory can still derive optimal emission reduction targets under these conditions, ${ }^{1}$ policy makers strongly prefer situations in which success of a policy is immediate, visible, and certain. A pragmatic way to define optimal policy is to rely on the internationally agreed target of maximum average warming of two degrees Celsius. Given the maximum temperature increase, climate physics has calculated the maximally allowed emission flow. ${ }^{2}$ This allows to determine necessary emission reductions on a global level. But the efficient reduction of greenhouse gas emissions is only fixed for all the countries together. In order to allocate the burden of emission reduction to single countries, equity aspects like differentiated responsibility, capability, and fairness have to be considered. If this is not done in a broadly accepted manner, parties will ultimately not be willing to sign the climate treaty. It has been shown that the different designs for a climate agreement differ dramatically in terms of their distributional impact. ${ }^{3}$ One of the central tasks is to determine how the burden of climate policy efforts should be shared between developed and developing countries.

\footnotetext{
${ }^{1}$ See Bretschger and Vinogradova (2014) and Bretschger and Karydas (2013).

${ }^{2}$ Meinshausen et al. (2009).

${ }^{3}$ See Bretschger and Mollet (2015).
} 
To implement climate policy on a country level entails three major problems, a cost problem and two types of distributional issues. With regard to the cost, many integrated assessment models provide diverse results and often overstate the costs of climate policy, especially when static frameworks are used. In fact, the static effect of decreasing fossil fuel input is well known to be negative. But the long-run character of climate policy necessitates deriving costs of climate policy in a dynamic environment, considering growing economies. As growth is driven by capital accumulation and technical progress, the relationship between carbon emissions and the accumulation of capital and knowledge are crucial for the cost calculation. Recent empirical evidence suggests that physical and knowledge capital accumulation are partially crowded out by abundant energy use, see Bretschger (2015). Put differently, the prediction of "induced innovation" (Hicks 1932) suggests that decreasing energy use fosters additional innovation improving energy efficiency. Accordingly, a decrease in carbon emissions can have a favorable growth effect, which has to be combined with the static effect when properly calculating the costs of climate policies.

With regards to the distributional issues, climate policy affects income distribution on a national and an international scale. On the national level, climate policy in the form of carbon taxes or carbon permits has often been seen as being potentially unfair to the poor because of rising energy prices. However, it is the tax system in a country as a whole that has to be evaluated to see whether it is equitable and fair. On an international level, in both cases of climate action and climate inaction, world income distribution is heavily affected. Lacking or insufficient climate policy would exert the biggest negative effects on climate-vulnerable and poorer economies, which would ultimately increase the inequalities in world income distribution. On the contrary, active climate policies are perceived by the developed countries as being disproportionately costly, if they are designed in a Kyoto-like manner. Specifically, some developed countries fear the loss of competitiveness of their energy-intensive industries, if other countries do not commit to substantial climate policies. A good produced by their domestic firms becomes relatively more expensive as the costs of production rise when emissions taxes have to be paid or permits need to be purchased.

Any climate agreement ultimately assigns specific shares of the global carbon budget to each individual country. For the design of an efficient policy, the total carbon budget needs to be compatible with the 2 degrees Celsius target. ${ }^{4}$ As regards the budget distribution, let us start with two rather extreme proposals. The first is labeled "Equal access to carbon space" and consists of dividing the global carbon budget equally to each inhabitant of the planet, see BASIC (2011). Using this egalitarian criterion it emerges that developed countries have already used up almost their entire carbon budget up to now or even exceeded it. Conversely, the LDCs have still abundant carbon budget to be used in the coming decades. The second proposal is to implement a unique global carbon price through carbon taxes where each country is entitled to keep full tax revenues, see Weitzmann (2014) domestic. Here the result for burden sharing is that the carbon intensive developed countries receive a

\footnotetext{
${ }^{4}$ Meinshausen et al. (2009) calculate carbon budgets of 1040 GT to 1440 GT CO2 for 2000-2050.
} 
large carbon budget, because they have large tax revenues, while the carbon extensive LDCs get a small part, because they generate low tax revenues, see Bretschger and Mollet (2015). A third approach using several basic equity principles has been put forward by Bretschger (2013). The equity-based proposal allocates the carbon budget according to four basic equity principles:

- Ability to pay: The larger is the economic capacity of a country, the more a country should contribute,

- Cost sharing: The lower are the costs of climate policy in a country, the more a country should contribute,

- Technical contribution: The more a country has achieved in advancing carbonefficient technologies, the less a country has to contribute

- Technical development: The lower was the range of technical alternatives to fossil fuels in energy conversion at the time of fossil use, the less carbon emissions are weighted for a country's responsibility.

When applying the equity-based approach we get an allocation of the global budget which lies between the two extreme proposals of the egalitarian approach and the unique carbon tax. On the one hand, developed countries obtain a lower budget than with the unified price approach mainly because of their economic capacity and historic responsibility. On the other hand, LDCs receive a somewhat lower budget than with the egalitarian approach, mainly because of the broad range of renewable energies which have been developed over the last years. The principle for technical development appears to be important because, in order to find a fair solution, one has to compare like with like; this includes the technology options at the time of the emissions. Interestingly, if the principle of technology development is ignored and the other principles are weighted equally we are back to the egalitarian proposal of BASIC (2011).

The final decision for the allocation of the global carbon budget is subject to international negotiations, science can only provide guidelines derived from accepted first principles. Climate policy in the form of tradeable emission permits has a clear advantage over national carbon taxes. However, financial globalization and the Great Recession generated high volatility of asset prices, including the price of emission certificates, so that the noise of the price signal has been fairly high. Hence, the decision whether we limit emission quantities or set carbon prices has also to consider the stringency of the regulation of international financial markets. What will definitely be a novelty is that LDCs will be part of a global social climate contract, which requires them to restrict future carbon emission. Even if the climate budget is not yet binding today, it will become binding in the future. Redirecting development to a less carbon-intensive economy entails economic adjustments which may conflict with unconstrained economic development. The failure of LDCs participation in international climate policy would repeat the failure of the Kyoto Protocol to cover only a decreasing share of world emissions and, moreover, raise the political risk of developed countries to impose import tariffs. However, the "only sticks" approach, 
possibly involving trade sanctions, is not appropriate in the case of LDCs, for the following reasons:

- it harms the right to development significantly, as foreign trade is an important engine of growth,

- it is not based on equity and fairness because LDCs did not contribute to the climate problem in the past,

- it is not efficient as future growth of the world economy will be mainly effectuated by the emerging economies and the LDCs,

- it affects foreign investors from developed countries by decreasing the profitability of their subsidiaries in the LDCs,

- it may turn out not to be feasible, as it could fail to comply with WTO rules; according to WTO agreement, trade provisions should be preceded by major efforts to negotiate with partners within a reasonable timeframe.

Proposed measures to enforce and strengthen an international climate agreement should thus not only include "sticks" but also "carrots", as in the Montreal Protocol of 1987 or "clean development mechanism", where trade measures were accompanied by financing arrangements and technology transfers. This is especially warranted in the case of LDCs. If emissions rise up to the assigned budget limit one can label this pattern as the LDC's "right to development." Developing countries, however, will have to demonstrate a "meaningful" commitment (Zhang 2009), i.e., they are not required to comply with environmental regulations immediately but should take some actions towards compliance at some future date. This is akin to the "grace" period granted to LDCs under the Montreal protocol. Moreover, if foreign aid is granted in the form of technical aid, growth of the LDCs can be supported and the so-called "aid curse," the crowding out of domestic growth forces, can be avoided. If an adequate system is implemented, it can also be used for international emission trading between developed countries and LDCs, in case there is no global emission trading scheme, which is very likely. The institutions performing these tasks are already established. The mandate of the powerful Green Climate Fund (GCF) is precisely to provide support to developing countries in order to limit or reduce their greenhouse gas emissions and to adapt to the impacts of climate change while the Least Developed Countries Fund for Climate Change (LDCF) is already operating but still not adequately funded. Of course one cannot neglect the often difficult institutional environment in LDCs, impeding maximum efficiency in controlling environmental achievements and potentially promoting rent-seeking activities when investments are subsidized or aid is given and distributed. The paper focuses on optimal environmental policies and abstracts from institutional frictions for the sake of conciseness.

The following sections develop formally the sets of conditions which should be satisfied for less developed economies voluntarily accepting to equitably participate in global climate policy by restricting future carbon emissions. The basic model idea is that international transfer compensate the welfare losses of climate policies in the LDCs. 


\section{Theoretical framework}

\subsection{Unconstrained economy}

Consider an LDC economy which produces a single consumption good using capital, according to the production function $Q_{t}=A K_{t}$, where $A>0$ is a technology parameter. Output can be either consumed or invested. As a by-product of production and consumption processes, greenhouse gas (GHG) emissions are released into the atmosphere. A technology for reducing the emissions problem exists. It requires capital investment, with the effectiveness of emissions control being positively related to the stock of equipment utilized for that purpose.

Thus the flow of emissions $E$ at time $t$ is given by

$$
E_{t}=\phi_{c} c_{t}+\phi_{k} K_{t}-\phi_{x} X_{t}+\bar{E},
$$

where $c_{t}$ stands for consumption, $K_{t}$ for physical capital stock, $X_{t}$ is the economy's stock of emissions control capital and $\phi_{c}, \phi_{k}$, and $\phi_{x}$ are positive constants (assumed to be less than unity) which measure pollution intensity of consumption, pollution intensity of physical capital, and abatement intensity of pollution-control capital, respectively. As can be seen from Eq. 1, consumption and capital become less carbon intensive with increasing $X$, which is a very general and convenient representation of abatement activities in an economy. The parameter $\bar{E}$ stands for global pollution; for a single LDC it is taken as given. Without loss of generality it will be normalized to zero in the rest of the analysis.

The economy is inhabited by an infinitely-lived representative individual who derives utility from consumption and disutility from pollution according to the additively-separable utility function $u\left(c_{t}\right)=\ln c_{t}-\frac{1}{2} E_{t}^{2}$. In the unconstrained economy, there is no climate policy in place. The country does not optimize over $E_{t}$ but is just maximizing utility from consumption with the aim to increase living standards. Thus in the unconstrained economy we have $X_{t}=0$ since there is neither an official abatement policy nor an incentive for private agents to undertake climate mitigation activities. The objective is

$$
\max _{c_{t}} \int_{0}^{\infty} u\left(c_{t}, E_{t}\right) e^{-\rho t} d t,
$$

subject to the standard physical capital accumulation constraint

$$
\dot{K}_{t}=Q\left(K_{t}\right)-c_{t}, \quad K_{0} \quad \text { given. }
$$

The rate of time preference is a constant $\rho$. The solution to this standard problem (see Appendix for derivation) is characterized by the path of consumption as

$$
\frac{\dot{c_{t}}}{c_{t}} \equiv \hat{c_{t}}=A-\rho .
$$

This is the standard Keynes-Ramsey growth rate equal to the difference between the marginal product of capital and the pure rate of time preference, i.e., given the assumed log-preferences and the absence of environmental policy. The time profile 
of consumption is then $c_{t}=c_{0} e^{(A-\rho) t}$. Substituting the latter expression into the capital accumulation constraint (3) yields:

$$
\dot{K}_{t}=A K_{t}-c_{0} e^{(A-\rho) t}
$$

and thus, using the transversality condition, $c_{0}=K_{0} / \rho$. Knowing the initial consumption rate, the time path of the physical capital stock can be completely characterized. It turns out that consumption, capital stock, and emissions (see Eq. 1) grow at the same rate $A-\rho$, so that $K_{t}=K_{0} e^{(A-\rho) t}$ and $E_{t}=\left(\frac{\phi_{c}}{\rho}+\phi_{k}\right) K_{0} e^{(A-\rho) t}$. The present discounted value of lifetime welfare is then given by

$$
W=\int_{0}^{\infty}\left[\ln c_{t}-\frac{1}{2} E_{t}^{2}\right] e^{-\rho t} d t=\frac{\ln c_{0}}{\rho}+\frac{A-\rho}{\rho^{2}}-\left(\frac{\phi_{c}}{\rho}+\phi_{k}\right)^{2} \frac{K_{0}^{2}}{2} \frac{1}{2 A-3 \rho} .
$$

This welfare level will constitute an imported benchmark for the evaluation of the climate policies introduced next.

\subsection{Climate policy}

We now examine the optimal behavior of the economy when climate policy is introduced. Below we consider two types of regulation, which combine the commitment of LCDs to a climate policy with a specific reward.

\subsubsection{Type I regulation}

Type I regulation requires a country to limit its emissions to a given level $\varepsilon$ by a given date $\tau$. The reduction in emissions (or a constrained increase in emissions) must follow a pre-specified plan. The notion of "meaningful commitment" of the LDC is captured in the model by the constant $\theta$, which is the requested rate of emissions decline (in the case of increasing emissions $-\theta$ is the rate of allowed emission increase, where naturally we have $-\theta<A-\rho$ ). From time $\tau$ onwards emissions must not exceed the predefined level $\varepsilon$. If the economy complies with the regulation, it will receive a flow of aid (or monetary compensation) equal to the amount $F$ on day $\tau$ and subsequently $F e^{-g(t-\tau)}$, i.e., the compensation will be decreasing at the rate $g .5$

We assume that the technology for producing the specific pollution-control equipment is not available in the LDC. Rather it must be imported from abroad at the price $P$ per unit, with the consumption good being the numeraire. The pollution-control capital is accumulated in a standard way:

$$
\dot{X}_{t}=I_{t}, \quad X_{0} \text { given, }
$$

where $I_{t}$ is the investment rate in pollution control.

Suppose the economy wishes to comply with the regulation. Then its optimal programme will consist of two phases: Phase I which lasts from time 0 to time $\tau$,

\footnotetext{
${ }^{5}$ The decline of the flow of aid in time can be rationalized by the limited commitment of the advanced countries but also by the development process in the less advanced countries.
} 
and Phase II which lasts from $\tau$ onwards. Let us first analyze Phase II. The control variables pertaining to Phase II are marked by a tilde.

Phase II the detailed derivation is relegated to the Appendix, while here we present only the relevant equations. The growth rate of consumption is given by:

$$
\frac{\dot{\tilde{c}}_{t}}{\tilde{c}_{t}}=\frac{\gamma \phi_{x}}{P}-\rho \equiv \psi=>\tilde{c}_{t}=\tilde{c}_{\tau} e^{\psi(t-\tau)},
$$

where $\gamma \equiv \frac{A P}{\phi_{x}+P \phi_{k}}$ and the initial consumption rate of Phase II is:

$$
\tilde{c}_{\tau}=\left(K_{\tau}+\frac{\tilde{F}}{\psi+\rho+g}\right) \frac{\rho}{\delta_{c}},
$$

where $\tilde{F} \equiv \frac{F \phi_{x}}{\phi_{x}+P \phi_{k}}, \delta_{c} \equiv \frac{\phi_{x}+P \phi_{c} \psi}{\phi_{x}+P \phi_{k}}$ and $K_{\tau}$ is the capital stock inherited from Phase I.

Phase I the growth rate of consumption in Phase I is the same as in Phase II:

$$
\frac{\dot{c}_{t}}{c_{t}}=\frac{\gamma \phi_{x}}{P}-\rho \equiv \psi=>c_{t}=c_{0} e^{\psi t},
$$

The time path of the physical capital stock can be obtained as:

$$
K_{t}=K_{0} e^{(\psi+\rho) t}-c_{0} \delta_{c} \frac{e^{(\psi+\rho) t}-e^{\psi t}}{\rho}-\delta_{\varepsilon} \frac{e^{(\psi+\rho) t}-e^{-\theta t}}{\theta+\psi+\rho},
$$

where $\delta_{\varepsilon} \equiv \frac{P \theta \varepsilon \varepsilon^{\theta \tau}}{\phi_{x}+P \phi_{k}}$. Combining the solutions for the consumption paths in both phases with the path of the capital stock allows us to obtain the optimal initial consumption rate:

$$
c_{0}^{I}=\frac{\rho}{\delta_{c}}\left[K_{0}-\delta_{\varepsilon} \frac{1-e^{-(\psi+\rho+\theta) \tau}}{\theta+\psi+\rho}+\frac{\tilde{F} e^{-(\psi+\rho) \tau}}{\psi+\rho+g}\right] .
$$

The superscript "I" stands for Type I regulation. The initial consumption rate depends positively on the initial stock of physical capital, $K_{0}$, the flow of aid promised to the country in the case of compliance, $F$, and the effectiveness of pollution control equipment, $\phi_{x}$. It depends negatively on the imposed emissions threshold, $\varepsilon$, the compliance date, $\tau$, the intensity of emissions stemming from consumption process, $\phi_{c}$, the imposed rate of emissions decline, $\theta$, the rate of decline in aid flow, $g$, and finally on the price of pollution-control equipment, $P$ (if $\tau$ is sufficiently long or $K_{0}$ sufficiently small).

Knowing $c_{0}^{I}$, the present value of lifetime welfare can be obtained:

$$
W^{I}=\frac{\ln c_{0}^{I}}{\rho}+\frac{\psi}{\rho^{2}}-\frac{\varepsilon^{2}\left(\rho e^{2 \theta \tau}+2 \theta e^{-\rho \tau}\right)}{2 \rho(2 \theta+\rho)} .
$$

\subsubsection{Type II regulation}

Similarly to the first policy, Type II regulation states that the country must reduce its emissions to a given level $\varepsilon$ by a given date $\tau$. Again, the emissions reduction must follow a prespecified plan such that the rate of emissions decline must be equal to a 
given constant $\theta$ (or the increase must equal $-\theta$ ). From time $\tau$ onwards emissions must not exceed $\varepsilon$. The new element is that, if the economy complies with the regulation, it will start receiving a flow of aid (or monetary compensation) which is tied to the investment in pollution control $F\left(\tilde{I}_{t}\right)>0$ with $F^{\prime}\left(\tilde{I}_{t}\right)>0$. Thus the flow of aid is not declining over time, as in Type I regulation, but is conditional on abatement effort. This scheme is effectively identical to a subsidy on purchases of pollutioncontrol equipment, although the LDC becomes eligible for the subsidy only once it has complied with the regulation deadline.

Assume, for simplicity, that $F^{\prime}(\tilde{I})$ is equal to a positive constant $\sigma$, i.e., the aid to LDC is proportional to its investment in pollution control. Then we obtain the growth rate of consumption as

$$
\hat{\tilde{c}}=\frac{A \phi_{x}}{\phi_{k}(P-\sigma)+\phi_{x}}-\rho \equiv \tilde{\psi}>\psi .
$$

The last inequality holds because $\psi=\frac{A \phi_{x}}{P \phi_{k}+\phi_{x}}-\rho$. Therefore, under Type II regulation, when the aid is conditional on the investment in pollution control, the growth rate of consumption in the second phase (when the regulation is binding) is higher than under Type I regulation, where aid is unconditional.

Following similar steps as in the previous subsection, we have:

$$
\dot{K}_{t}=(\tilde{\psi}+\rho) K_{t}-\tilde{\delta}_{c} \tilde{c}_{\tau} e^{\tilde{\psi}(t-\tau)},
$$

where $\tilde{\delta}_{c}=\frac{\phi_{x}+(P-\sigma) \phi_{c} \tilde{\psi}}{\phi_{x}+(P-\sigma) \phi_{k}}$. Integrating the above differential equation from $\tau$ to infinity and applying the transversality condition allows to solve for the initial consumption rate of Phase II:

$$
\tilde{c}_{\tau}=\frac{\rho K_{\tau}}{\tilde{\delta}_{c}}=\frac{\rho K_{\tau}\left[\phi_{x}+(P-\sigma) \phi_{k}\right]}{\phi_{x}+(P-\sigma) \phi_{c} \tilde{\psi}},
$$

and $K_{\tau}$ is the capital stock inherited from Phase I. Since the LDC's optimal program in Phase I under Type II regulation is identical to the one under Type I regulation, we already have the solution for $K_{\tau}$ from the previous subsection. Hence we can solve directly for the initial consumption rate in Phase I:

$$
c_{0}^{I I}=\frac{\rho e^{\rho \tau}}{\tilde{\delta}_{c}+\delta_{c}\left(e^{\rho \tau}-1\right)}\left[K_{0}-\delta_{\varepsilon} \frac{1-e^{-(\rho+\psi+\theta) \tau}}{\theta+\psi+\rho}\right]
$$

The present value of lifetime welfare under Type II regulation is given by:

$$
W^{I I}=\frac{\ln c_{0}^{I I}}{\rho}+\frac{\psi+\tilde{\psi} e^{-\rho \tau}}{\rho^{2}}-\frac{\varepsilon^{2}\left(\rho e^{2 \theta \tau}+2 \theta e^{-\rho \tau}\right)}{2 \rho(2 \theta+\rho)}
$$

Having solved for the lifetime welfare under the two regulation types, we are now in the position to analyze the conditions such that an LDC chooses to comply with the first or the second regulation or not to comply at all. 


\section{Analysis of compliance}

The LDC will chose to voluntarily participate in global climate policy, i.e. to comply with the proposed regulation, if and only if its lifetime welfare under compliance is at least as large as its welfare under non-compliance. The policy thus respects the LDC's "right to development."

The policy tools at the disposal of the regulators from the world community are:

- $\quad$ emissions threshold $\varepsilon$

- emissions decline rate $\theta$

- compliance deadline $\tau$

- pollution-control subsidy $\sigma$

- compensation $F$

The model also embeds the possibility of a technology transfer from the advanced to the developing countries by affecting $\phi_{c}, \phi_{k}$ and $\phi_{x}$. The questions we want to address in the following are: What type of regulation the developing country is more likely to comply with? Under what conditions? Which tools are more effective in inducing compliance? Do countries' characteristics, such as initial capital stocks, rate of time preference, polluting and abating intensities, etc., matter for the compliance? If yes, then what type of regulation should be applied for what type of countries? Given that less advanced countries are not homogeneous in terms of their development levels, it is natural to think that different types of regulations should be designed for different groups of countries, expressing specific notions of differentiated responsibility.

At this stage we need to distinguish between two possible cases: (i) LDC must reduce its emissions, $\theta>0$; and (ii) LDC is allowed to increase its emissions (but at the prespecified rate and only up to the threshold $\varepsilon$ ), $\theta<0$. In order to get the full picture of the compliance issue it is necessary to work through the different model cases; this will allow us to clearly focus our discussion of the relevant policy conclusions in the following sections.

\subsection{Case (i): emissions reduction, $\theta>0$}

\subsubsection{Type I regulation vs status quo}

This section examines the conditions that should be in place so that LDC complies voluntarily with Type I regulation instead of choosing the unconstrained development, i.e. the status quo (hereafter SQ). In particular, we look at the combinations of the emissions threshold $\varepsilon$ and the rate of emissions decline, $\theta$, such that LDC is indifferent between the two options, i.e., $W^{I}=W$. We define the difference between the two welfare levels as $D^{I} \equiv W^{I}-W$, so that

$$
D^{I}=\frac{\ln c_{0}^{I}-\ln c_{0}}{\rho}-\frac{\varepsilon^{2}\left(\rho e^{2 \theta \tau}+2 \theta e^{-\rho \tau}\right)}{2 \rho(2 \theta+\rho)}+\frac{\psi-A+\rho}{\rho^{2}}-\frac{K_{0}^{2}}{2}\left(\frac{\phi_{c}}{\rho}+\phi_{k}\right)^{2} \frac{1}{2 A-3 \rho} .
$$


Setting $D^{I}$ to zero defines a schedule in $\varepsilon$ and $\theta$ space along which LDC is indifferent between Type I regulation and SQ. The slope of the schedule is given by (see Appendix for exact expression)

$$
\left.\frac{d \theta}{d \varepsilon}\right|_{D^{I}=0}=-\frac{\partial D^{I} / \partial \varepsilon}{\partial D^{I} / \partial \theta}=-\frac{\partial W^{I} / \partial \varepsilon}{\partial W^{I} / \partial \theta}<0 .
$$

The sign of the expression above hinges on the fact that $\theta>0$ in this case. Thus the $D^{I}=0$ schedule is negatively sloped. Interestingly enough, a smaller emissions target must be accompanied by a slower convergence rate in order to keep an LDC indifferent between complying with Type I regulation and Status Quo. This is because emissions enter negatively LDC's utility function and thus the requirement to reduce emissions $(\theta>0)$ is beneficial for LDC in terms of welfare.

\subsubsection{Type II regulation vs status quo}

Similarly, define the difference between the welfare levels under Type II regulation and SQ as $D^{I I} \equiv W^{I I}-W$ :

$$
D^{I I}=\frac{\ln c_{0}^{I I}-\ln c_{0}}{\rho}+\frac{\tilde{\psi} e^{-\rho \tau}+\psi-A+\rho}{\rho^{2}}-\frac{\varepsilon^{2}\left(\rho e^{2 \theta \tau}+2 \theta e^{-\rho \tau}\right)}{2 \rho(2 \theta+\rho)}-\frac{K_{0}^{2}}{2}\left(\frac{\phi_{c}}{\rho}+\phi_{k}\right)^{2} \frac{1}{2 A-3 \rho} .
$$

The slope of the $D^{I I}=0$ schedule is given by

$$
\left.\frac{d \theta}{d \varepsilon}\right|_{D^{I I}=0}=-\frac{\partial D^{I I} / \partial \varepsilon}{\partial D^{I I} / \partial \theta}=-\frac{\partial W^{I I} / \partial \varepsilon}{\partial W^{I I} / \partial \theta}<0 .
$$

Thus the $D^{I I}=0$ schedule is negatively sloped: a smaller emissions target must be accompanied by a slower convergence rate in order to keep an LDC indifferent between complying with Type II regulation and Status Quo. It can be shown that the $D^{I I}=0$ schedule is flatter than the $D^{I}=0$ schedule (see Appendix).

\subsubsection{Type I vs type II regulation}

Under what conditions an LDC is more likely to comply with one or the other type of regulation? The answer to this question depends on how the LDC's welfare is affected by various policies under the two regulations. Let us define the difference in lifetime welfare under regulation II $\left(W^{I I}\right)$ and regulation $\mathrm{I}\left(W^{I}\right)$ by $D$, i.e.,

$$
D \equiv W^{I I}-W^{I}=\frac{\ln c_{0}^{I I}-\ln c_{0}^{I}}{\rho}+\frac{\tilde{\psi} e^{-\rho \tau}}{\rho^{2}} .
$$

Clearly, when $c_{0}^{I I}>c_{0}^{I}$, the difference in welfare is positive, so that an LDC will always choose to comply with Type II regulation but not with Type I. For the rest of the analysis we continue to assume that the initial conditions are such that $c_{0}^{I I}<c_{0}^{I}$. 
We are interested in combinations of $\theta$ and $\varepsilon$ such that $D=0$. The slope of the $D=0$ schedule is given by

$$
\begin{gathered}
\left.\frac{d \theta}{d \varepsilon}\right|_{D=0}=-\frac{\partial D / \partial \varepsilon}{\partial D / \partial \theta}=-\frac{\partial W^{I I} / \partial \varepsilon-\partial W^{I} / \partial \varepsilon}{\partial W^{I I} / \partial \theta-\partial W^{I} / \partial \theta} \\
=-\frac{\frac{1}{c_{0}^{I I}} \frac{\partial c_{0}^{I I}}{\partial \varepsilon}-\frac{1}{c_{0}^{I}} \frac{\partial c_{0}^{I}}{\partial \varepsilon}}{\frac{1}{c_{0}^{I I}} \frac{\partial c_{0}^{I I}}{\partial \theta}-\frac{1}{c_{0}^{I}} \frac{\partial c_{0}^{I}}{\partial \theta}}=-\frac{\frac{\partial c_{0}^{I}}{\partial \varepsilon}\left(\frac{1}{c_{0}^{I I}} \mu-\frac{1}{c_{0}^{I}}\right)}{\frac{\partial c_{0}^{I}}{\partial \theta}\left(\frac{1}{c_{0}^{I I}} \mu-\frac{1}{c_{0}^{I}}\right)}=-\frac{\frac{\partial c_{0}^{I}}{\partial \varepsilon}}{\frac{\partial c_{0}^{I}}{\partial \theta}}<0 .
\end{gathered}
$$

It can be shown that the $D=0$ schedule is flatter than the $D^{I I}=0$ schedule (see Appendix).

The relative positions of the three schedules are illustrated graphically in Fig. 1. The schedules divide the quadrant into six zones. Each zone is characterized by the combinations of $\theta$ and $\varepsilon$ such that one of the three options, i.e., the Status Quo or Type I regulation or Type II regulation, dominates the other two. An increase in $\varepsilon$ has a negative effect on $W^{I}$ and $W^{I I}$ and no effect on the status quo welfare. Thus, $W>W^{I}$ to the right of $D^{I}=0$ and $W>W^{I I}$ to the right of $D^{I I}=0$. We also know that an increase in $\varepsilon$ has a more negative effect on $W^{I I}$ than on $W^{I}$ and hence $W^{I}>W^{I I}$ above and to the right of $D=0$. Thus the six zones can be grouped in three: (i) the zone of compliance with Type I regulation (hereafter $Z C^{I}$ ), (ii) the zone of compliance with Type II regulation (hereafter $Z C^{I I}$ ), and (iii) the zone of non-compliance (hereafter $Z N C$ ), as illustrated in Fig. 2a. Type I regulation is preferred when the emissions target, $\varepsilon$, is relatively low while the convergence rate, $\theta$, is moderate. Type II regulation is preferred for a wide range of emissions threshold but with the convergence rate being faster (slower) the higher (lower) the threshold.

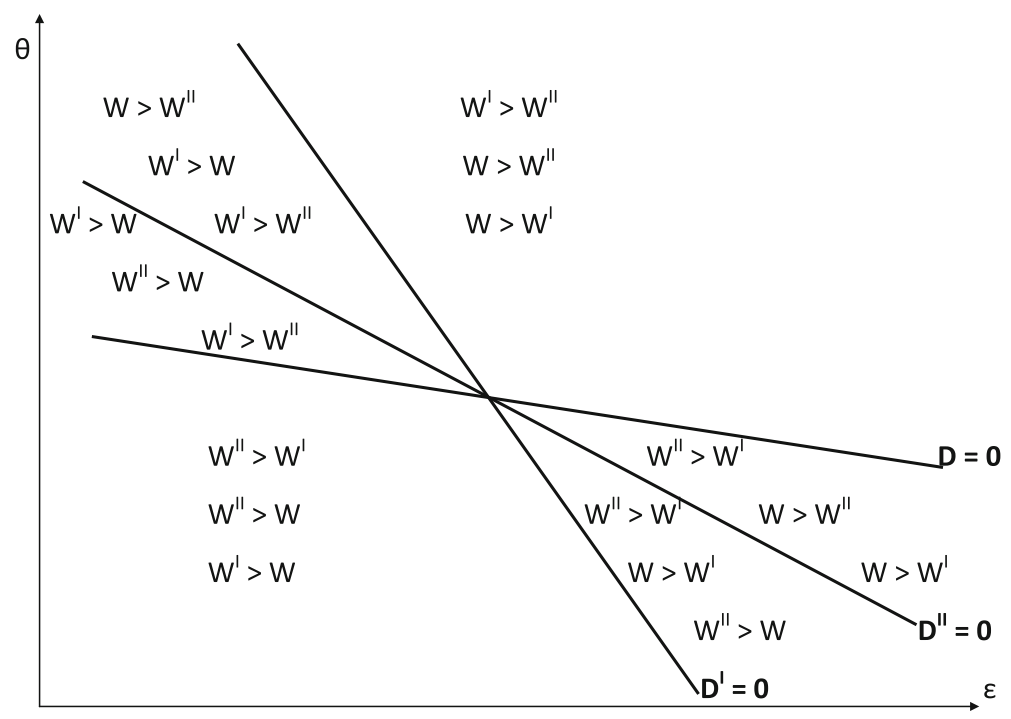

Fig. 1 Emissions threshold and emissions reduction speed, $\theta>0$ 


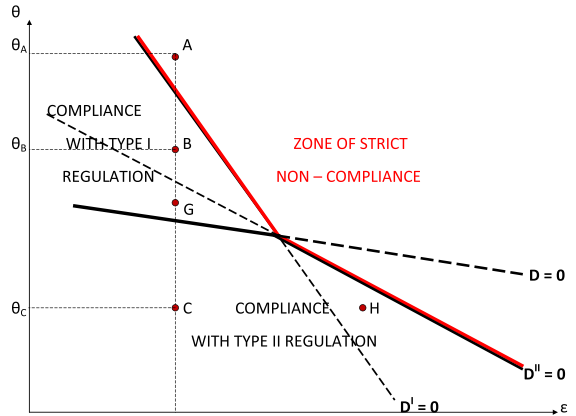

a

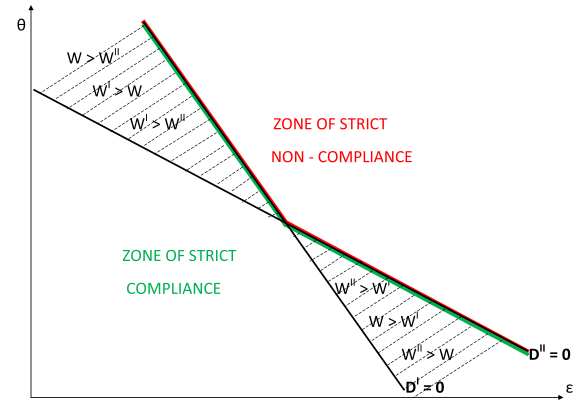

b

Fig. 2 Zones of compliance and non-compliance, $\theta>0$

The non-compliance is preferred when either the convergence rate is relatively high and the emissions target relatively low or when both are relatively high. This latter case arises when the emissions target imposed by a regulation is in fact above the emissions level attained by a non-regulated economy. This situation is not relevant for our further analysis.

Consider, for instance, points like A, B, and C in Fig. 2a, which are all located at the same targeted emissions level. Depending on the proposed convergence rate, an LDC will either choose not to comply with any regulation (if $\theta$ is relatively high, such as $\theta_{A}$ ), or to comply with Type I regulation (if $\theta$ is relatively moderate, such as $\theta_{B}$ ), or to comply with Type II regulation (if $\theta$ is relatively low, such as $\theta_{C}$ ). Voluntary compliance with Type II regulation requires a slower convergence rate, $\theta$, because the (negative) effect of $\theta$ on $W^{I I}$ (working through the consumption rate) is stronger than on $W^{I}$. Thus, for any targeted emissions threshold, the choice of the convergence speed determines which regulation type will be voluntarily accepted by an LDC.

Consider next a point in the zone of compliance with Type I regulation such as $\mathrm{G}$. Assume that the combination of $\theta$ and $\varepsilon$ corresponding to point $\mathrm{G}$ (which lies in $Z C^{I}$ ) is proposed within the Type II regulation but Type I is not offered. Will an LDC still comply? The answer is yes, because for this combination of $\theta$ and $\varepsilon, W^{I I}$ exceeds $W$, as can be seen in Fig. 1. If, however, the combination B is proposed, then an LDC will choose not to comply since $W^{I I}$ falls short of $W$ for the corresponding $\theta$ and $\varepsilon$ (see Fig. 1). More generally, for any combination of $\theta$ and $\varepsilon$ which lies between $D^{I}=0$ and $D^{I I}=0$ to the left of their intersection, an LDC will prefer noncompliance if only Type II regulation is offered. Similarly, for any combination of $\theta$ and $\varepsilon$ which lies between $D^{I}=0$ and $D^{I I}=0$ to the right of their intersection (such as, e.g., point $\mathrm{H}$ ), an LDC will choose not to comply if Type I is the only regulation available. If, however, $\theta$ and $\varepsilon$ lie between $D=0$ and $D^{I I}=0$ to the left of their intersection, belonging to $Z C^{I}$, but only Type II regulation is offered, then an LDC will still choose to comply. And finally, for any combinations of $\theta$ and $\varepsilon$ which fall below $D=0$ and to the left of $D^{I}=0$ an LDC will voluntarily comply, regardless of whether the regulation is of Type I or Type II. This zone will be referred to as Zone of Strict Compliance (see Fig. 2b). 


\subsection{Case (ii): emissions increase, $\theta<0$}

Numerous developing countries claim their right to increase emissions in the coming years, although with a commitment to stick to a given schedule. This is the situation we wish to analyze in this section. Instead of reducing emissions to a threshold $\varepsilon$ by the date $\tau$, an LDC is allowed to increase its emissions at the rate $|\theta|$ until they reach $\varepsilon$ by the date $\tau$ and subsequently emissions should not exceed $\varepsilon$.

The analysis proceeds along similar lines as presented in the previous section. The key difference is that now, since $\theta<0$, the slopes of all the three schedules become positive, although their relative position does not change. The change in the slope sign occurs because, with $\theta<0, \frac{\partial c_{0}^{I}}{\partial \varepsilon}>0$ and thus $\frac{\partial W^{I}}{\partial \varepsilon}>0$ when the initial conditions are such that $\frac{1}{c_{0}} \frac{\partial c_{0}^{I}}{\partial \varepsilon}>\varepsilon \frac{\rho e^{-2|\theta| \tau}-2|\theta| e^{-\rho \tau}}{\rho-2|\theta|}$. This occurs, for example, when the initial capital stock $K_{0}$ is small, the promised aid flow $F$ is small or the price of antipollution equipment $P$ is relatively large.

Figure 3 illustrates the three schedules and shows that they divide the $|\theta|$ and $\varepsilon$ space into six areas. In each area, the combination of emission growth rate and emissions threshold is such that one of the three options dominates the other two. The zones of compliance and non-compliance are depicted in Fig. 4. For instance, Fig. 4a shows that if the emissions growth rate is allowed to be relatively high (high $|\theta|$ ) and the emissions ceiling is relatively large (high $\varepsilon$ ), then an LDC prefers to comply with Type I regulation. If the emissions requirement is strict (low $\varepsilon$ ), however, then an LDC prefers non-compliance, regardless the emissions growth rate. Type II regulation is preferred when the emission threshold regulation is relatively loose and the convergence rate is moderately high. Figure $4 \mathrm{~b}$ demonstrates that an LDC will

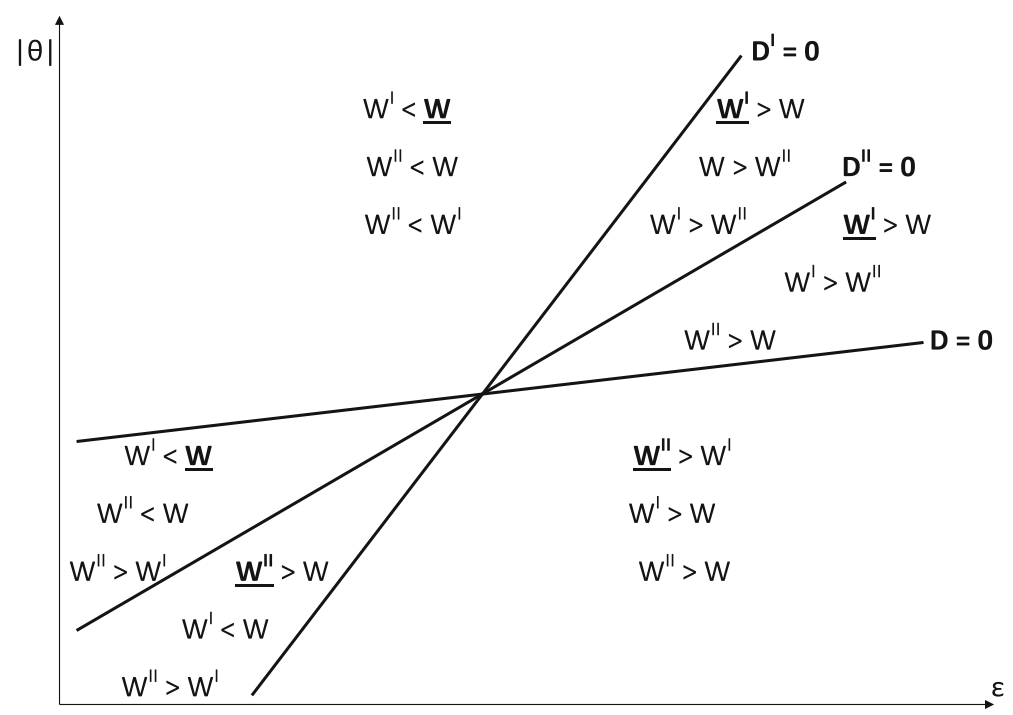

Fig. 3 Emissions threshold and emissions reduction speed, $\theta<0$ 


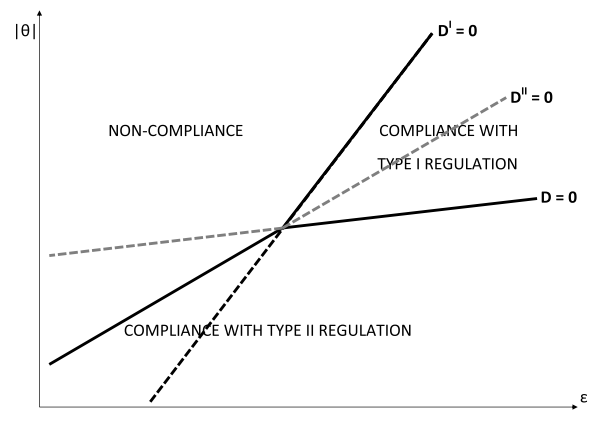

a

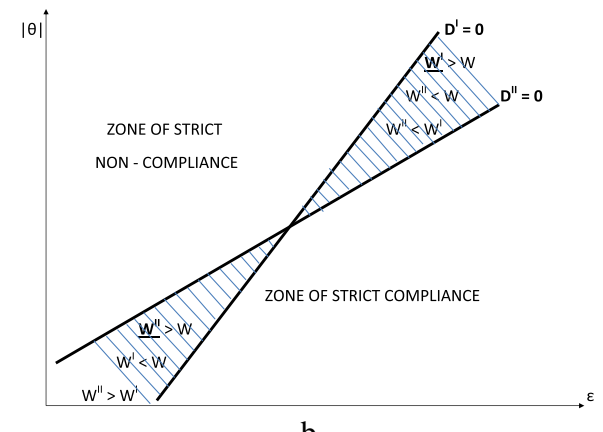

$\mathrm{b}$

Fig. 4 Zones of compliance and non-compliance, $\theta<0$

choose compliance, regardless of which regulation type is offered with a wide range of emissions growth rates, if the emissions threshold is not too strict. This area is marked as "zone of strict compliance". On the other hand, there are two zones, shaded by the thin lines, where a specific regulation type is dominated by both SQ and the other regulation type. Consider the top right-hand shaded area. The combinations of $|\theta|$ and $\varepsilon$ are such that $W^{I}>W, W^{I}>W^{I I}$ but $W^{I I}<W$. If these combinations of policy instruments are proposed but only Type II regulation is offered, an LDC will not comply. If, by contrast, Type I regulation is offered or both regulations are offered, an LDC will choose to comply with Type I. The opposite occurs in the bottom left-hand shaded area, where the emissions threshold is relatively strict and convergence rates are moderate. In this case $W^{I I}>W, W^{I I}>W^{I}$ but $W^{I}<W$. Consequently, if only Type I regulation is on the table, an LDC will choose non-compliance.

\section{Policy analysis}

In this section we explore how a change in the incentives to comply affects the relative position of the zones of compliance and non-compliance. We focus on the amount of aid $F$ and anti-pollution subsidy $\sigma$. We only consider the case of declining emissions $(\theta>0)$. The case of increasing emissions can be analyzed along the same lines and the qualitative conclusions are similar.

\subsection{Unconditional aid, $F$}

In the present framework, the unconditional foreign aid, or a monetary compensation, is the amount $F$ given to LDC on date $\tau$ if compliance with Type I regulation is achieved. During the subsequent periods, i.e., $t>\tau$, LDC receives $F e^{-g(t-\tau)}$, where $g$ is the rate at which the foreign aid declines over time. As mentioned earlier, this decline in the amount of monetary transfer may reflect the limited commitment on behalf of donors or gradual improvement in the standard of living in LDC due to 


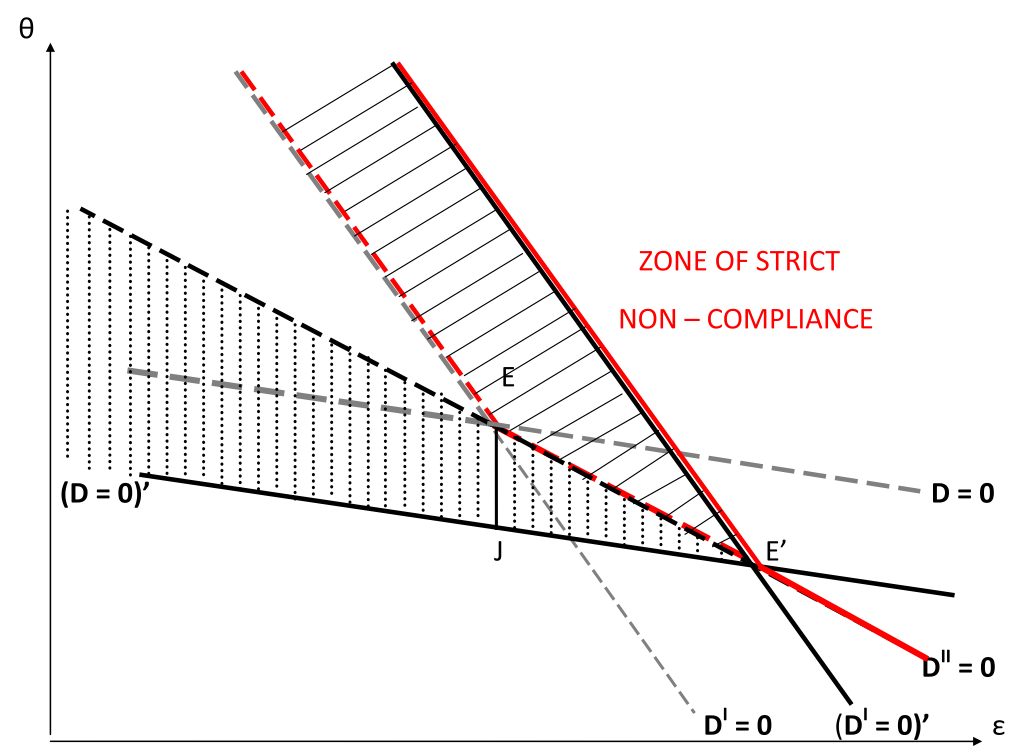

Fig. 5 Increase in foreign aid

development process. ${ }^{6}$ The unconditional aid, $F$, affects only the lifetime welfare $W^{I}$ and has no effect on either $W^{I I}$ or $W$. A higher $F$ unambiguously improves $W^{I}$ through it's positive effect on the initial consumption rate $c_{0}^{I}$ :

$$
\frac{\partial c_{0}^{I}}{\partial F}=\frac{\rho \phi_{x} e^{-(\psi+\rho) \tau}}{\left(\phi_{x}+P \psi \phi_{c}\right)(\psi+\rho+g)}>0 .
$$

This induces a rightward shift of the $D^{I}=0$ schedule and a downward shift of the $D=0$ schedule (see Fig. 3). The magnitudes of the respective (horizontal) shifts are given by

$$
\left.\frac{d \varepsilon}{d F}\right|_{D^{I}=0}=-\frac{\frac{1}{c_{0}^{I}} \frac{\partial c_{0}^{I}}{\partial F}}{\frac{1}{c_{0}^{I}} \frac{\partial c_{0}^{I}}{\partial \varepsilon}-\frac{\varepsilon\left(\rho e^{2 \theta \tau}+2 \theta e^{-\rho \tau}\right)}{2 \theta+\rho}}>0
$$

and

$$
\left.\frac{d \varepsilon}{d F}\right|_{D=0}=-\frac{-\frac{1}{c_{0}^{I}} \frac{\partial c_{0}^{I}}{\partial F}}{\frac{1}{c_{0}^{I I}} \frac{\partial c_{0}^{I I}}{\partial \varepsilon}-\frac{1}{c_{0}^{I}} \frac{\partial c_{0}^{I}}{\partial \varepsilon}}<0 .
$$

\footnotetext{
${ }^{6}$ The qualitative results do not depend on the sign of $g$. It can be positive, as in our assumption in the text, or it can be zero (constant flow of aid) or even negative (increasing flow of aid over time). $g$ only affects the initial consumption rate of the economy: $\frac{\partial c_{0}^{I}}{\partial g}=\frac{\rho \widetilde{F} e^{-(\psi+r h o) \tau}}{\delta_{c}(\psi+\rho+g)^{2}}<0$, so that a zero or a negative $g$ would imply a higher consumption rate and thus a higher present value of lifetime welfare under Type I regulation. The obvious consequence is that compliance with Type I regulation in this case becomes more likely, as the $D^{I}=0$ schedule shifts outwards. Note that $g$ does not affect the slope of the schedule.
} 


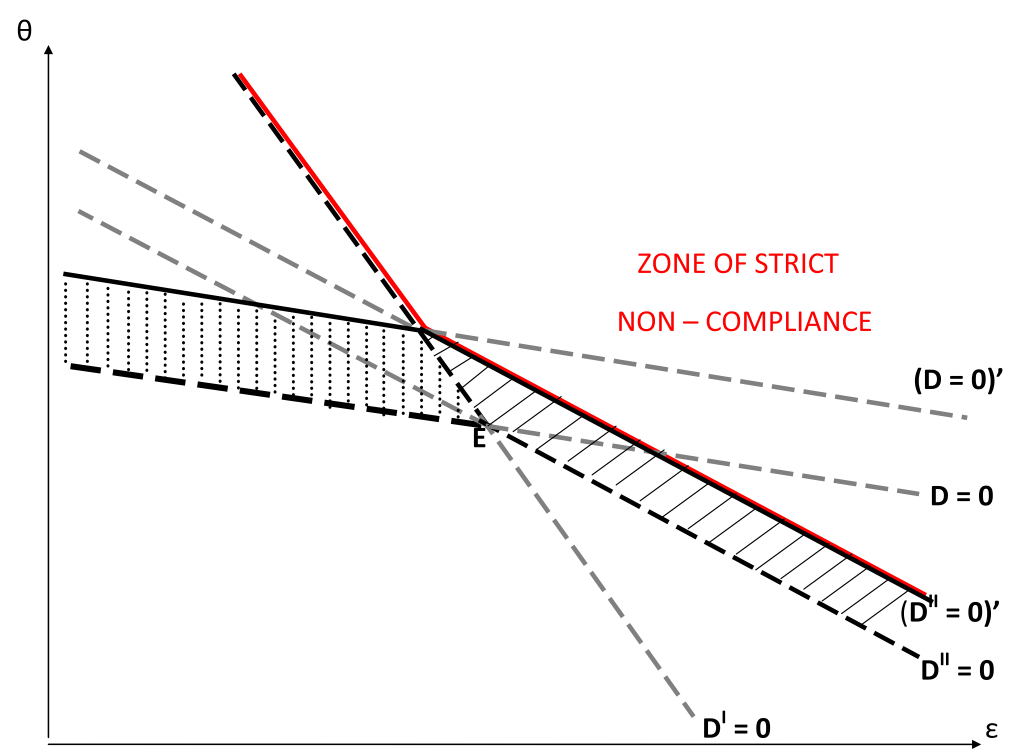

Fig. 6 Increase in pollution-control subsidy

The dashed lines in Fig. 5 represent the original equilibrium, while the solid lines labeled $\left(D^{I}=0\right)^{\prime}$ and $(D=0)^{\prime}$ are drawn for a higher value of $F$. The overall effect of the policy is to expand the zone of compliance with Type I regulation $\left(Z C^{I}\right)$ at the expense of the zone of strict non-compliance $(Z N C)$, shaded by the slanted solid lines, and the zone of compliance with Type II regulation $\left(Z C^{I I}\right)$, shaded by the vertical dotted lines. When a higher amount of foreign aid is promised in case of compliance with Type I regulation, an LDC is willing to accept a wider range of convergence rates and emission thresholds. These include faster convergence rates for the same emissions-target level, as in the area between $D^{I}=0$ and $\left(D^{I}=0\right)^{\prime}$ to the left of $E J$ line, but also faster convergence rates accompanied by a less stringent emissions target, as in the area $E J^{\prime} J$.

\subsection{Pollution-control subsidy, $\sigma$}

The pollution-control subsidy, $\sigma$, affects only $W^{I I}$ and hence induces shifts of $D^{I I}=$ 0 and $D=0$, while $D^{I}=0$ schedule is not affected. The horizontal shift of $D^{I I}=0$ is given by:

$$
\left.\frac{d \varepsilon}{d \sigma}\right|_{D^{I I}=0}=-\frac{\frac{1}{c_{0}^{I I}} \frac{\partial c_{0}^{I I}}{\partial \sigma}+\frac{e^{-\rho \tau}}{\rho} \frac{\partial \tilde{\psi}}{\partial \sigma}}{\frac{1}{c_{0}^{I I}} \frac{\partial c_{0}^{I I}}{\partial \varepsilon}-\frac{\varepsilon\left(\rho e^{2 \theta \tau}+2 \theta e^{-\rho \tau}\right)}{2 \theta+\rho}},
$$

where the denominator is unambiguously negative (as has been shown earlier), while we show in the Appendix that the numerator is positive. Thus, the $D^{I I}=0$ schedule shifts to the right when $\sigma$ increases. 
The horizontal shift of $D=0$ schedule is given by:

$$
\left.\frac{d \varepsilon}{d \sigma}\right|_{D^{I I}=0}=-\frac{\frac{1}{c_{0}^{I I}} \frac{\partial c_{0}^{I I}}{\partial \sigma}+\frac{e^{-\rho \tau}}{\rho} \frac{\partial \tilde{\psi}}{\partial \sigma}}{\frac{1}{c_{0}^{I I}} \frac{\partial c_{0}^{I I}}{\partial \varepsilon}-\frac{1}{c_{0}^{I}} \frac{\partial c_{0}^{I}}{\partial \varepsilon}}>0,
$$

since the numerator is positive and the denominator is negative (see Appendix). Thus, the $D=0$ schedule shifts up and to the right when $\sigma$ increases. This is illustrated graphically in Fig. 6. The total effect of the policy (i.e., an increase in the pollutioncontrol subsidy) is to expand the zone of compliance with Type II regulation at the expense of $Z C^{I}$ (shaded by dotted vertical lines) and $Z N C$ (shaded by solid slanted lines). Consequently both $Z C^{I}$ and $Z N C$ shrink. With a higher $\sigma$, an LDC is willing to comply with the Type II regulation characterized by faster convergence rates for any given emissions target.

\section{Conclusion}

There is a global agreement that efforts should be made to deal with climate change. However, there is not yet an agreement on how these efforts should be shared between advanced and developing countries. Advanced economies fear the loss of competitiveness of their domestic firms when the latter must purchase pollution permits in order to comply with environmental standards. Developing countries prioritize economic growth and improvement in the standard of living over environmental problems. Moreover, they refer to the right to development granted by international agreements. This paper derives efficient and equitable rules for global burden sharing in climate policy and especially looks at voluntary climate policy by LDCs in terms of compliance with environmental regulation. It studies the perspective of a developing country and examines the constellations of conditions and policies that should be in place in order to guarantee voluntary compliance.

We focus on supporting and stimulating measures provided by the advanced countries to the developing country, such as monetary transfers and anti-pollution equipment subsidy. More specifically, we analyze two types of regulation: One where a predefined transfer is initiated on the date of compliance with emissions target; and the other where the amount transferred is tied to emissions-control effort. Both regulations, however, impose an emissions target that should be achieved by a given date and the rate of convergence to this target. We distinguish between two scenarios. In the first scenario, an LDC must follow a plan of emissions reduction. In the second scenario, an LDC is allowed to have increasing emissions over time until they reach a given threshold by a given date. We show the combinations of the emissions target and the convergence rate such that the country is willing to comply with either the first or the second regulation type or does not comply at all. We find that, first, offering one or the other option is inefficient. The chances that an LDC complies voluntarily with environmental standards are higher when a menu of options is on the table. The direct implication of this results is that the number and/or diversity of countries willing to comply with environmental standards is 
also higher when a variety of alternatives is available instead of just one regulation type. Second, the policy mix, in terms of emissions threshold and convergence rate, must be carefully designed depending not only on the regulation type (conditional or unconditional compensation) but also on whether a strict reduction in emissions is required or (constrained) emissions growth is allowed. The latter scenario applies to least-developed economies with very low per-capita carbon intensity. Finally, an improvement in compliance incentives - be they unconditional or conditional unambiguously increases the chances of reaching a successful climate agreement.

\section{Appendix}

\section{A Unconstrained economy}

The current-value Hamiltonian associated with the optimization program can be written as

$$
H=u\left(c_{t}, E_{t}\right)+\lambda_{t}\left[Q\left(K_{t}\right)-c_{t}\right] .
$$

The optimality conditions are (time subscripts are suppressed for notational convenience):

$$
\begin{aligned}
& c: \frac{\partial u}{\partial c}-\lambda=0, \\
& K: \lambda Q^{\prime}(K)=\rho \lambda-\dot{\lambda},
\end{aligned}
$$

and the transversality condition $\lim _{t \rightarrow \infty} K_{t} e^{-\rho t}=0$. It follows from Eqs. A.1-A.2 that $\frac{\dot{c}}{c} \equiv \hat{c}=A-\rho$.

\section{B Type I regulation solution}

Phase II The optimization problem is to

$$
\max _{\tilde{c}_{t}} \int_{\tau}^{\infty} u\left(\tilde{c}_{t}, \varepsilon\right) e^{-\rho t} d t
$$

subject to

$$
\begin{aligned}
& \dot{K}_{t}=Q\left(K_{t}\right)-\tilde{c}_{t}-P \tilde{I}_{t}+F e^{-g(t-\tau)}, \\
& \dot{X}_{t}=\tilde{I}_{t} \\
& \phi_{c} \tilde{c}_{t}+\phi_{k} K_{t}-\phi_{x} X_{t}=\varepsilon
\end{aligned}
$$

where a tilde over a control variable indicates that the variable pertains to Phase II. The Hamiltonian may be written as:

$$
H=u\left(\tilde{c}_{t}, \varepsilon\right)+\lambda_{t}\left[Q\left(K_{t}\right)-\tilde{c}_{t}-\tilde{I}_{t} P+F e^{-g(t-\tau)}\right]+\mu_{t} \tilde{I}_{t}+\eta_{t}\left[\phi_{c} \tilde{c}_{t}+\phi_{k} K_{t}-\phi_{x} X_{t}-\varepsilon\right]
$$


The optimality conditions are (time subscripts are suppressed for notational convenience):

$$
\begin{aligned}
\tilde{c} & : \frac{\partial u}{\partial \tilde{c}}-\lambda+\eta \phi_{c}=0, \\
\tilde{I} & :-\lambda P+\mu=0, \\
K & : \lambda Q^{\prime}(K)+\eta \phi_{k}=\rho \lambda-\dot{\lambda}, \\
X & :-\eta \phi_{x}=\rho \mu-\dot{\mu},
\end{aligned}
$$

and the transversality condition $\lim _{t \rightarrow \infty} K_{t} e^{-\rho t}=0$. The optimality conditions imply that

$$
\frac{\dot{\tilde{c}}}{\tilde{c}}=\frac{\gamma \phi_{x}}{P}-\rho \equiv \psi=>\tilde{c}_{t}=\tilde{c}_{\tau} e^{\psi(t-\tau)},
$$

so that consumption grows at the rate $\psi$, assumed positive. Since emissions are constrained by the environmental regulation, the two capital stocks must be related as:

$$
X_{t}=\frac{1}{\phi_{x}}\left[\phi_{k} K_{t}+\phi_{c} \tilde{c}_{\tau} e^{\psi(t-\tau)}-\varepsilon\right]
$$

and thus the investment rate in pollution control is given by

$$
\tilde{I}_{t}=\dot{X}_{t}=\frac{1}{\phi_{x}}\left[\phi_{k} \dot{K}_{t}+\psi \phi_{c} \tilde{c}_{\tau} e^{\psi(t-\tau)}\right] .
$$

Using this in Eq. A.3 yields:

$$
\dot{K}_{t}=(\psi+\rho) K_{t}-\tilde{c}_{\tau} \frac{\phi_{x}+P \phi_{c} \psi}{\phi_{x}+P \phi_{k}} e^{\psi(t-\tau)}+\frac{F \phi_{x}}{\phi_{x}+P \phi_{k}} e^{-g(t-\tau)} .
$$

Integrating the above differential equation from $\tau$ to infinity and applying the transversality condition allows to solve for the initial consumption rate of Phase II:

$$
\tilde{c}_{\tau}=\left(K_{\tau}+\frac{\tilde{F}}{\psi+\rho+g}\right) \frac{\rho}{\tilde{\delta}_{c}}
$$

where $\tilde{F} \equiv \frac{F \phi_{x}}{\phi_{x}+P \phi_{k}}$ and $\tilde{\delta}_{c} \equiv \frac{\phi_{x}+P \phi_{c} \psi}{\phi_{x}+P \phi_{k}}$ and $K_{\tau}$ is the capital stock inherited from Phase I to which we now turn.

Phase I The optimization problem is to

$$
\max _{c_{t}} \int_{0}^{\tau} u\left(c_{t}, \varepsilon e^{\theta(\tau-t)}\right) e^{-\rho t} d t
$$

subject to

$$
\begin{aligned}
& \dot{K}_{t}=Q\left(K_{t}\right)-c_{t}-P I_{t}, \\
& \dot{X}_{t}=I_{t}, \\
& \phi_{c} c_{t}+\phi_{k} K_{t}-\phi_{x} X_{t}=\varepsilon e^{\theta(\tau-t)} .
\end{aligned}
$$

The Hamiltonian is then

$$
H=u\left(c_{t}, \varepsilon e^{\theta(\tau-t)}\right)+\lambda_{t}\left[Q\left(K_{t}\right)-c_{t}-P I_{t}\right]+\mu_{t} I_{t}+\eta_{t}\left[\phi_{c} c_{t}+\phi_{k} K_{t}-\phi_{x} X_{t}-\varepsilon e^{\theta(\tau-t)}\right]
$$


and the first-order conditions

$$
\begin{aligned}
c & : \frac{\partial u}{\partial c}-\lambda+\eta \phi_{c}=0, \\
I & :-\lambda P+\mu=0, \\
K & : \lambda Q^{\prime}(K)+\eta \phi_{k}=\rho \lambda-\dot{\lambda}, \\
X & :-\eta \phi_{x}=\rho \mu-\dot{\mu} .
\end{aligned}
$$

This set of conditions allows to solve for the growth rate of consumption in Phase I:

$$
\frac{\dot{c}}{c}=\frac{\gamma \phi_{x}}{P}-\rho \equiv \psi=>c_{t}=c_{0} e^{\psi t},
$$

so that consumption grows at the same rate $\psi$ in both phases. Then, using Eqs. A.16 and A.14, the time path of the physical capital stock can be obtained:

$$
K_{t}=K_{0} e^{(\psi+\rho) t}-\delta_{c} \frac{e^{(\psi+\rho) t}-e^{\psi t}}{\rho}-\delta_{\varepsilon} \frac{e^{(\psi+\rho) t}-e^{-\theta t}}{\theta+\psi+\rho},
$$

where $\delta_{\varepsilon} \equiv \frac{P \theta \varepsilon \varepsilon^{\theta \tau}}{\phi_{x}+P \phi_{k}}$ and $\delta_{c} \equiv \frac{c_{0}\left(\phi_{x}+P \psi \phi_{c}\right)}{\phi_{x}+P \phi_{k}}$ is defined as before. Since consumption grows continuously at the same rate in both phases, we have $\tilde{c}_{\tau}=c_{\tau}=c_{0} e^{\psi \tau}$. We can therefore combine (7) and (A.22), evaluated at time $t=\tau$, to solve for the optimal initial consumption rate:

$$
c_{0}^{I}=\frac{\rho}{\phi_{x}+P \psi \phi_{c}}\left[K_{0}\left(\phi_{x}+P \phi_{k}\right)-\frac{P \theta \varepsilon\left(e^{\theta \tau}-e^{-(\psi+\rho) \tau}\right)}{\theta+\psi+\rho}+\frac{F \phi_{x} e^{-(\psi+\rho) \tau}}{\psi+\rho+g}\right] .
$$

Knowing $c_{0}^{I}$, the present value of lifetime welfare can be obtained:

$$
\begin{aligned}
W^{I} & =\int_{0}^{\tau} u\left(c_{t}, \varepsilon e^{\theta(\tau-t)}\right) e^{-\rho t} d t+\int_{\tau}^{\infty} u\left(\tilde{c}_{t}, \varepsilon\right) e^{-\rho t} d t \\
& =\int_{0}^{\infty} \ln \left(c_{0}^{I} e^{\psi t}\right) e^{-\rho t} d t-\int_{0}^{\tau} \frac{1}{2}\left(\varepsilon e^{\theta(\tau-t)}\right)^{2} e^{-\rho t} d t-\int_{\tau}^{\infty} \frac{1}{2} \varepsilon^{2} e^{-\rho t} d t \\
& =\int_{0}^{\infty} \ln c_{0}^{I} e^{-\rho t} d t+\int_{0}^{\infty} \psi t e^{-\rho t} d t-\frac{1}{2} \varepsilon^{2} \int_{0}^{\tau} e^{2 \theta(\tau-t)-\rho t} d t-\frac{1}{2} \varepsilon^{2} \int_{\tau}^{\infty} e^{-\rho t} d t \\
& =\frac{\ln c_{0}^{I}}{\rho}-\left.\psi\left[\frac{e^{-\rho t}}{\rho}\left(t+\frac{1}{\rho}\right)\right]\right|_{0} ^{\infty}-\frac{\varepsilon^{2} e^{2 \theta \tau}}{2} \int_{0}^{\tau} e^{-(2 \theta+\rho) t} d t-\frac{\varepsilon^{2}}{2} \int_{\tau}^{\infty} e^{-\rho t} d t \\
& =\frac{\ln c_{0}^{I}}{\rho}+\frac{\psi}{\rho^{2}}-\frac{\varepsilon^{2}\left(\rho e^{2 \theta \tau}+2 \theta e^{-\rho \tau}\right)}{2 \rho(2 \theta+\rho)},
\end{aligned}
$$

where the superscript "I" stands for "compliance with Type I regulation".

\section{Type II regulation solution}

The Hamiltonian associated with Phase II optimization program may be written as:

$H=u\left(\tilde{c}_{t}, \varepsilon\right)+\lambda_{t}\left[Q\left(K_{t}\right)-\tilde{c}_{t}-\tilde{I}_{t} P+F\left(\tilde{I}_{t}\right)\right]+\mu_{t} \tilde{I}_{t}+\eta_{t}\left[\phi_{c} \tilde{c}_{t}+\phi_{k} K_{t}-\phi_{x} X_{t}-\varepsilon\right]$. 
The optimality conditions are (time subscripts are suppressed for notational convenience):

$$
\begin{aligned}
\tilde{c} & : \frac{\partial u}{\partial \tilde{c}}-\lambda+\eta \phi_{c}=0, \\
\tilde{I} & : \lambda\left[F^{\prime}(\tilde{I})-P\right]+\mu=0, \\
K & : \lambda Q^{\prime}(K)+\eta \phi_{k}=\rho \lambda-\dot{\lambda}, \\
X & :-\eta \phi_{x}=\rho \mu-\dot{\mu},
\end{aligned}
$$

and the transversality condition $\lim _{t \rightarrow \infty} K_{t} e^{-\rho t}=0$.

Assume, for simplicity, that $F^{\prime}(\tilde{I})$ is equal to a positive constant $\sigma$, i.e., the aid to LDC is proportional to its investment in pollution control. Then, from Eq. A.27, we have $\mu=(P-\sigma) \lambda$ and thus $\hat{\mu}=\hat{\lambda}$. Dividing (A.28) by $\lambda,($ A.29) by $\mu$, and equating the resulting equations, we obtain $\frac{\eta}{\lambda}=\frac{A(\sigma-P)}{\phi_{x}-\phi_{k}(\sigma-P)} \equiv \tilde{\gamma}$. Using this in Eq. A.28 yields a constant growth rate of $\lambda$, i.e., $\hat{\lambda}=\rho-A-\tilde{\gamma} \phi_{k}$. Combining this with Eq. A.26, we obtain the growth rate of consumption as

$$
\hat{\tilde{c}}=\frac{A \phi_{x}}{\phi_{k}(P-\sigma)+\phi_{x}}-\rho \equiv \tilde{\psi}>\psi .
$$

The last inequality holds because $\psi=\frac{A \phi_{x}}{P \phi_{k}+\phi_{x}}-\rho$. Therefore, under Type II regulation, when the aid is conditional on the investment in pollution control, the growth rate of consumption in the second phase (when the regulation is binding) is higher than under Type I regulation, where aid is unconditional.

Following similar steps as in the previous subsection, we have:

$$
X_{t}=\frac{1}{\phi_{x}}\left[\phi_{k} K_{t}+\phi_{c} \tilde{c}_{\tau} e^{\tilde{\psi}(t-\tau)}-\varepsilon\right]
$$

and thus the investment rate in pollution control is given by

$$
\tilde{I}_{t}=\dot{X}_{t}=\frac{1}{\phi_{x}}\left[\phi_{k} \dot{K}_{t}+\tilde{\psi} \phi_{c} \tilde{c}_{\tau} e^{\tilde{\psi}(t-\tau)}\right] .
$$

Using this in Eq. A.3 yields:

$$
\dot{K}_{t}=(\tilde{\psi}+\rho) K_{t}-\tilde{\delta}_{c} \tilde{c}_{\tau} e^{\tilde{\psi}(t-\tau)},
$$

where $\tilde{\delta}_{c}=\frac{\phi_{x}+(P-\sigma) \phi_{c} \tilde{\psi}}{\phi_{x}+(P-\sigma) \phi_{k}}$. Integrating the above differential equation from $\tau$ to infinity and applying the transversality condition allows to solve for the initial consumption rate of Phase II:

$$
\tilde{c}_{\tau}=\frac{\rho K_{\tau}}{\tilde{\delta}_{c}}=\frac{\rho K_{\tau}\left[\phi_{x}+(P-\sigma) \phi_{k}\right]}{\phi_{x}+(P-\sigma) \phi_{c} \tilde{\psi}},
$$

and $K_{\tau}$ is the capital stock inherited from Phase I. Since the LDC's optimal program in Phase I under Type II regulation is identical to the one under Type I regulation, we already have the solution for $K_{\tau}$ from the previous subsection. Evaluating (A.22) at 
$t=\tau$ and equating with $K_{\tau}$ expressed in terms of $\tilde{c}_{\tau}$ from Eq. A.34, we can solve for the initial consumption rate in Phase I:

$$
c_{0}^{I I}=\frac{\rho\left[K_{0} e^{\rho \tau}-\delta_{\varepsilon} \frac{e^{\rho \tau}-e^{-(\theta+\psi) \tau}}{\theta+\psi+\rho}\right]}{\frac{\phi_{x}+(P-\sigma) \phi_{c} \tilde{\psi}}{\phi_{x}+(P-\sigma) \phi_{k}}+\frac{\left(\phi_{x}+P \psi \phi_{c}\right)\left(e^{\rho \tau}-1\right)}{\phi_{x}+P \phi_{k}}}
$$

or, substituting for $\delta_{\varepsilon}$,

$$
c_{0}^{I I}=\frac{\rho\left[K_{0} e^{\rho \tau}\left(\phi_{x}+P \phi_{k}\right)-\frac{\left(e^{\theta \tau}-e^{-(\rho+\psi) \tau}\right) P \theta \varepsilon e^{\rho \tau}}{(\theta+\psi+\rho)}\right]\left[\phi_{x}+(P-\sigma) \phi_{k}\right]}{\left[\phi_{x}+(P-\sigma) \phi_{c} \tilde{\psi}\right]\left(\phi_{x}+P \phi_{k}\right)+\left[\phi_{x}+(P-\sigma) \phi_{k}\right]\left(\phi_{x}+P \psi \phi_{c}\right)\left(e^{\rho \tau}-1\right)}
$$

The present value of lifetime welfare under Type II regulation is given by:

$$
W^{I I}=\frac{\ln c_{0}^{I I}}{\rho}+\frac{\psi+\tilde{\psi} e^{-\rho \tau}}{\rho^{2}}-\frac{\varepsilon^{2}\left(\rho e^{2 \theta \tau}+2 \theta e^{-\rho \tau}\right)}{2 \rho(2 \theta+\rho)}
$$

\section{Analysis of the slopes}

\section{D.1 Slope of $D^{I}=0$ schedule}

$$
D^{I}=\frac{\ln c_{0}^{I}-\ln c_{0}}{\rho}-\frac{\varepsilon^{2}\left(\rho e^{2 \theta \tau}+2 \theta e^{-\rho \tau}\right)}{2 \rho(2 \theta+\rho)}+\frac{\psi-A+\rho}{\rho^{2}} .
$$

The numerator is unambiguously negative:

$$
\frac{\partial W^{I}}{\partial \varepsilon}=\frac{1}{\rho c_{0}^{I}} \frac{\partial c_{0}^{I}}{\partial \varepsilon}-\varepsilon \frac{\rho e^{2 \theta \tau}+2 \theta e^{-\rho \tau}}{\rho(2 \theta+\rho)}<0,
$$

where

$$
\frac{\partial c_{0}^{I}}{\partial \varepsilon}=-\frac{\rho P \theta\left(e^{\theta \tau}-e^{-(\psi+\rho) \tau}\right)}{\left(\phi_{x}+P \psi \phi_{c}\right)(\theta+\psi+\rho)}<0 \text { for } \tau>0 .
$$

The denominator is also negative:

$$
\frac{\partial W^{I}}{\partial \theta}=\frac{1}{\rho c_{0}^{I}} \frac{\partial c_{0}^{I}}{\partial \theta}-\frac{\varepsilon^{2}\left[e^{2 \theta \tau}[\tau(2 \theta+\rho)-1]+e^{-\rho \tau}\right]}{(2 \theta+\rho)^{2}}<0,
$$

since

$$
\frac{\partial c_{0}^{I}}{\partial \theta}=-\frac{\rho P \varepsilon\left[e^{\theta \tau} \tau \theta^{2}+(\psi+\rho)\left[e^{\theta \tau}(1+\tau \theta)-e^{-(\psi+\rho) \tau}\right]\right]}{\left(\phi_{x}+P \psi \phi_{c}\right)(\theta+\psi+\rho)^{2}}<0
$$

and

$$
e^{2 \theta \tau}[\tau(2 \theta+\rho)-1]+e^{-\rho \tau}>0 \text { for } \tau>0
$$




\section{D.1 Slope of $D^{I I}=0$ schedule}

$$
D^{I I}=\frac{\ln c_{0}^{I I}-\ln c_{0}}{\rho}+\frac{\tilde{\psi} e^{-\rho \tau}+\psi-A+\rho}{\rho^{2}}-\frac{\varepsilon^{2}\left(\rho e^{2 \theta \tau}+2 \theta e^{-\rho \tau}\right)}{2 \rho(2 \theta+\rho)} .
$$

The slope of the $D^{I I}=0$ schedule is given by

$$
\left.\frac{d \theta}{d \varepsilon}\right|_{D^{I I}=0}=-\frac{\partial D^{I I} / \partial \varepsilon}{\partial D^{I I} / \partial \theta}=-\frac{\partial W^{I I} / \partial \varepsilon}{\partial W^{I I} / \partial \theta}<0 .
$$

The numerator is unambiguously negative:

$$
\frac{\partial W^{I I}}{\partial \varepsilon}=\frac{1}{\rho c_{0}^{I I}} \frac{\partial c_{0}^{I I}}{\partial \varepsilon}-\varepsilon \frac{\rho e^{2 \theta \tau}+2 \theta e^{-\rho \tau}}{\rho(2 \theta+\rho)}<0,
$$

where

$$
\frac{\partial c_{0}^{I I}}{\partial \varepsilon}=-\frac{\frac{\left(e^{\theta \tau}-e^{-(\rho+\psi) \tau}\right) P \theta \rho e^{\rho \tau}}{(\theta+\psi+\rho)}\left[\phi_{x}+(P-\sigma) \phi_{k}\right]}{\left[\phi_{x}+(P-\sigma) \phi_{c} \tilde{\psi}\right]\left(\phi_{x}+P \phi_{k}\right)+\left[\phi_{x}+(P-\sigma) \phi_{k}\right]\left(\phi_{x}+P \psi \phi_{c}\right)\left(e^{\rho \tau}-1\right)}<0 .
$$

We can also write

$$
\frac{\partial c_{0}^{I I}}{\partial \varepsilon}=\frac{\partial c_{0}^{I}}{\partial \varepsilon} \mu
$$

where

$$
\mu \equiv \frac{e^{\rho \tau}\left(\phi_{x}+P \psi \phi_{c}\right)\left[\phi_{x}+(P-\sigma) \phi_{k}\right]}{\left[\phi_{x}+(P-\sigma) \phi_{c} \tilde{\psi}\right]\left(\phi_{x}+P \phi_{k}\right)+\left[\phi_{x}+(P-\sigma) \phi_{k}\right]\left(\phi_{x}+P \psi \phi_{c}\right)\left(e^{\rho \tau}-1\right)}>0 .
$$

The denominator is also negative:

$$
\frac{\partial W^{I I}}{\partial \theta}=\frac{1}{\rho c_{0}^{I I}} \frac{\partial c_{0}^{I I}}{\partial \theta}-\frac{\varepsilon^{2}\left[e^{2 \theta \tau}[\tau(2 \theta+\rho)-1]+e^{-\rho \tau}\right]}{(2 \theta+\rho)^{2}}<0
$$

since

$$
\begin{gathered}
\frac{\partial c_{0}^{I I}}{\partial \theta}=-\frac{\frac{\rho P \varepsilon\left[\phi_{x}+(P-\sigma) \phi_{k}\right]\left\{e^{(\theta+\rho) \tau}\left[(\psi+\rho)(1+\tau \theta)+\tau \theta^{2}\right]-e^{-\psi \tau}(\psi+\rho)\right\}}{(\theta+\psi+\rho)^{2}}}{\left[\phi_{x}+(P-\sigma) \phi_{c} \tilde{\psi}\right]\left(\phi_{x}+P \phi_{k}\right)+\left[\phi_{x}+(P-\sigma) \phi_{k}\right]\left(\phi_{x}+P \psi \phi_{c}\right)\left(e^{\rho \tau}-1\right)} \\
=\frac{\partial c_{0}^{I}}{\partial \theta} \mu<0 .
\end{gathered}
$$




\section{D.3 Comparison of slopes of $D^{I}=0$ and $D^{I I}=0$ schedules}

By comparing the absolute values of the slopes, we need to prove that

$$
\begin{gathered}
\left.\left|\frac{d \theta}{d \varepsilon}\right|_{D^{I I}=0}|<| \frac{d \theta}{d \varepsilon}\right|_{D^{I}=0} \mid \\
\frac{\partial W^{I I} / \partial \varepsilon}{\partial W^{I I} / \partial \theta}<\frac{\partial W^{I} / \partial \varepsilon}{\partial W^{I} / \partial \theta} \\
\frac{1}{\rho c_{0}^{I I} \frac{\partial c_{0}^{I I}}{\partial \varepsilon}-\varepsilon \frac{\rho e^{2 \theta \tau}+2 \theta e^{-\rho \tau}}{\rho(2 \theta+\rho)}}<\frac{\frac{1}{\rho c_{0}^{I}} \frac{\partial c_{0}^{I}}{\partial \varepsilon}-\varepsilon \frac{\rho e^{2 \theta \tau}+2 \theta e^{-\rho \tau}}{\rho(2 \theta+\rho)}}{\frac{1}{\rho c_{0}^{I}} \frac{\partial c_{0}^{I}}{\partial \theta}-\frac{\varepsilon^{2}\left[e^{2 \theta \tau}[\tau(2 \theta+\rho)-1]+e^{-\rho \tau}\right]}{(2 \theta+\rho)^{2}}}
\end{gathered}
$$

For notational convenience define $y \equiv \frac{\rho e^{2 \theta \tau}+2 \theta e^{-\rho \tau}}{\rho(2 \theta+\rho)}$ and $z \equiv$ $\frac{\varepsilon^{2}\left[e^{2 \theta \tau}[\tau(2 \theta+\rho)-1]+e^{-\rho \tau}\right]}{(2 \theta+\rho)^{2}}$. Then we can rewrite the inequality as:

$$
\begin{gathered}
\frac{1}{\rho c_{0}^{I I} \frac{\partial c_{0}^{I I}}{\partial \varepsilon}-y} \frac{\frac{1}{\rho c_{0}^{I}} \frac{\partial c_{0}^{I}}{\partial \varepsilon}-y}{\frac{1}{\rho c_{0}^{I}} \frac{\partial c_{0}^{I}}{\partial \theta}-z} \\
\left(\frac{1}{\rho c_{0}^{I I}}-z \frac{\partial c_{0}^{I I}}{\partial c_{0}^{I I}}-y\right)\left(\frac{1}{\rho \varepsilon} \frac{\partial c_{0}^{I}}{\partial \theta}-z\right)<\left(\frac{1}{\rho c_{0}^{I I}} \frac{\partial c_{0}^{I I}}{\partial \theta}-z\right)\left(\frac{1}{\rho c_{0}^{I}} \frac{\partial c_{0}^{I}}{\partial \varepsilon}-y\right)
\end{gathered}
$$

multiplying the terms and recalling that $\frac{\partial c_{0}^{I I}}{\partial \varepsilon}=\frac{\partial c_{0}^{I}}{\partial \varepsilon} \mu$ and $\frac{\partial c_{0}^{I I}}{\partial \theta}=\frac{\partial c_{0}^{I}}{\partial \theta} \mu$ we obtain

$$
\begin{aligned}
\frac{1}{\rho c_{0}^{I I}} \frac{\partial c_{0}^{I}}{\partial \varepsilon} \mu \frac{1}{\rho c_{0}^{I}} \frac{\partial c_{0}^{I}}{\partial \theta} & -z \frac{1}{\rho c_{0}^{I I}} \frac{\partial c_{0}^{I}}{\partial \varepsilon} \mu-y \frac{1}{\rho c_{0}^{I}} \frac{\partial c_{0}^{I}}{\partial \theta}+y z<\frac{1}{\rho c_{0}^{I I}} \frac{\partial c_{0}^{I}}{\partial \theta} \mu \frac{1}{\rho c_{0}^{I}} \frac{\partial c_{0}^{I}}{\partial \varepsilon} \\
& -y \frac{1}{\rho c_{0}^{I I}} \frac{\partial c_{0}^{I}}{\partial \theta} \mu-z \frac{1}{\rho c_{0}^{I}} \frac{\partial c_{0}^{I}}{\partial \varepsilon}+y z .
\end{aligned}
$$

Eliminating identical terms on both sides and multiplying by $\rho$ we are left with

$$
z \frac{\partial c_{0}^{I}}{\partial \varepsilon}\left(\frac{1}{c_{0}^{I}}-\frac{1}{c_{0}^{I I}} \mu\right)<y \frac{\partial c_{0}^{I}}{\partial \theta}\left(\frac{1}{c_{0}^{I}}-\frac{1}{c_{0}^{I I}} \mu\right)
$$


Given that the term in the parentheses on the LHS is identical to the one on the RHS, we can divide through. However we need to keep in mind that this term is negative, so that division entails a change of the inequality sign. Then we have

$$
\begin{aligned}
& \Rightarrow \quad z \frac{\partial c_{0}^{I}}{\partial \varepsilon}>y \frac{\partial c_{0}^{I}}{\partial \theta} \\
& \Rightarrow-\frac{\varepsilon^{2}\left[e^{2 \theta \tau}[\tau(2 \theta+\rho)-1]+e^{-\rho \tau}\right]}{(2 \theta+\rho)^{2}} \frac{\rho P \theta\left(e^{\theta \tau}-e^{-(\psi+\rho) \tau}\right)}{\left(\phi_{x}+P \psi \phi_{c}\right)(\theta+\psi+\rho)} \\
& >-\frac{\rho P \varepsilon\left[e^{\theta \tau} \tau \theta^{2}+(\psi+\rho)\left[e^{\theta \tau}(1+\tau \theta)-e^{-(\psi+\rho) \tau}\right]\right]}{\left(\phi_{x}+P \psi \phi_{c}\right)(\theta+\psi+\rho)^{2}} \varepsilon \frac{\rho e^{2 \theta \tau}+2 \theta e^{-\rho \tau}}{\rho(2 \theta+\rho)} \\
& \Rightarrow-\frac{\left[e^{2 \theta \tau}[\tau(2 \theta+\rho)-1]+e^{-\rho \tau}\right] \theta\left(e^{\theta \tau}-e^{-(\psi+\rho) \tau}\right)}{2 \theta+\rho} \\
& >-\frac{\left(\rho e^{2 \theta \tau}+2 \theta e^{-\rho \tau}\right)\left[e^{\theta \tau}\left[\tau \theta^{2}+(\psi+\rho)(1+\tau \theta)\right]-(\psi+\rho) e^{-(\psi+\rho)}\right]}{\rho(\theta+\psi+\rho)}
\end{aligned}
$$

Multiplying both sides by $-(2 \theta+\rho) \rho(\theta+\psi+\rho)<0$ and noting that again the inequality will change sign, we get

$$
\begin{aligned}
& \Rightarrow \rho(\theta+\psi+\rho)\left[e^{2 \theta \tau}[\tau(2 \theta+\rho)-1]+e^{-\rho \tau}\right] \theta\left(e^{\theta \tau}-e^{-(\psi+\rho) \tau}\right) \\
& <(2 \theta+\rho)\left(\rho e^{2 \theta \tau}+2 \theta e^{-\rho \tau}\right)\left[e^{\theta \tau}\left[\tau \theta^{2}+(\psi+\rho)(1+\tau \theta)\right]-(\psi+\rho) e^{-(\psi+\rho)}\right] \\
& \Rightarrow e^{2 \theta \tau}\left\{\rho(\theta+\psi+\rho)[\tau(2 \theta+\rho)-1] \theta\left(e^{\theta \tau}-e^{-(\psi+\rho) \tau}\right)\right\} \\
& +e^{-\rho \tau}\left\{\rho(\theta+\psi+\rho) \theta\left(e^{\theta \tau}-e^{-(\psi+\rho) \tau}\right)\right\} \\
& <e^{2 \theta \tau}(2 \theta+\rho) \rho\left[e^{\theta \tau}\left[\tau \theta^{2}+(\psi+\rho)(1+\tau \theta)\right]-(\psi+\rho) e^{-(\psi+\rho)}\right] \\
& +e^{-\rho \tau}(2 \theta+\rho) 2 \theta\left[e^{\theta \tau}\left[\tau \theta^{2}+(\psi+\rho)(1+\tau \theta)\right]-(\psi+\rho) e^{-(\psi+\rho)}\right]
\end{aligned}
$$

Now compare the terms multiplying $e^{2 \theta \tau}$ on the LHS and the RHS:

$$
\begin{aligned}
& \Rightarrow \rho(\theta+\psi+\rho)[\tau(2 \theta+\rho)-1] \theta\left(e^{\theta \tau}-e^{-(\psi+\rho) \tau}\right) \\
& \sim(2 \theta+\rho) \rho\left[e^{\theta \tau}\left[\tau \theta^{2}+(\psi+\rho)(1+\tau \theta)\right]-(\psi+\rho) e^{-(\psi+\rho)}\right] \mid \div \rho \\
& \Rightarrow e^{\theta \tau}\left\{(\theta+\psi+\rho)[\tau(2 \theta+\rho)-1] \theta-(2 \theta+\rho)\left[\tau \theta^{2}+(\psi+\rho)(1+\tau \theta)\right]\right\} \\
& \sim e^{-(\psi+\rho)}\{(\theta+\psi+\rho)[\tau(2 \theta+\rho)-1] \theta-(2 \theta+\rho)(\psi+\rho)\}
\end{aligned}
$$

Define the term on the LHS as a function $\alpha(\tau)$ and the term on the RHS as a function $\beta(\tau)$. At $\tau=0$ we have $\alpha(0)=\beta(0)=-\theta(\theta+\psi+\rho)-(2 \theta+\rho)(\psi+\rho)$. The slopes are given by $\frac{d \alpha}{d \tau}=-[\theta(\theta+\psi+\rho)-(2 \theta+\rho)(\psi+\rho)] \theta e^{\theta \tau}<0$ and $\frac{d \beta}{d \tau}=$ $\{-(\psi+\rho)[(\theta+\psi+\rho)[\tau(2 \theta+\rho)-1] \theta-(2 \theta+\rho)(\psi+\rho)]+\theta(\theta+\psi+\rho)$ $(2 \theta+\rho)\} \times e^{-(\psi+\rho) \tau} \gtrless 0$. It can be shown that $\beta(\tau)$ is monotonically rising on 
$\tau \in\left[0, \tau^{*}\right)$, where $\tau^{*}=\frac{\theta(\theta+\psi+\rho)(2 \theta+\rho)+(\psi+\rho)[(2 \theta+\rho)(\psi+\rho)+\theta(\theta+\psi+\rho)]}{\theta(\theta+\psi+\rho)(2 \theta+\rho)(\psi+\rho)}>0$ is the maximum, and monotonically declining on $\tau \in\left(\tau^{*}, \infty\right)$. There is a unique $\bar{\tau}=\frac{\theta(\theta+\psi+\rho)+(2 \theta+\rho)(\psi+\rho)}{\theta(\theta+\psi+\rho)(2 \theta+\rho)}<\tau^{*}$ such that $\beta(\bar{\tau})=0$, a unique inflection point $\tilde{\tau}=\frac{2 \theta(\theta+\psi+\rho)(2 \theta+\rho)+(\psi+\rho)[(2 \theta+\rho)(\psi+\rho)+\theta(\theta+\psi+\rho)]}{\theta(\theta+\psi+\rho)(2 \theta+\rho)(\psi+\rho)}>\tau^{*}$, and $\lim _{\tau \rightarrow \infty} \beta(\tau)=0$. Given these characteristics, it is clear that $\alpha(\tau)<\beta(\tau) \forall \tau>0$. A similar analysis can be done for the terms multiplying $e^{-\rho \tau}$ to show that the term on the LHS is smaller than on the RHS. Thus we proved that the slope of $D^{I I}=0$ is smaller in absolute value than the slope of $D^{I}=0$.

\section{D.4 Comparison of slopes of $D=0$ and $D^{I I}=0$ schedules}

It can be shown the $D=0$ schedule is flatter than the $D^{I I}=0$ schedule:

$$
\begin{aligned}
& \left.\left|\frac{d \theta}{d \varepsilon}\right|_{D^{I I}=0}|<| \frac{d \theta}{d \varepsilon}\right|_{D^{I}=0} \mid \\
& \frac{\frac{1}{\rho c_{0}^{I I}} \frac{\partial c_{0}^{I I}}{\partial \varepsilon}-y}{\frac{1}{\rho c_{0}^{I I}} \frac{\partial c_{0}^{I I}}{\partial \theta}-z}>\frac{\frac{\partial c_{0}^{I}}{\partial \varepsilon}}{\frac{\partial c_{0}^{I}}{\partial \theta}} \\
& \frac{\frac{1}{\rho c_{0}^{I I}} \frac{\partial c_{0}^{I}}{\partial \varepsilon} \mu-y}{\frac{1}{\rho c_{0}^{I I}} \frac{\partial c_{0}^{I}}{\partial \theta} \mu-z}>\frac{\frac{\partial c_{0}^{I}}{\partial \varepsilon}}{\frac{\partial c_{0}^{I}}{\partial \theta}} \\
& \left(\frac{1}{\rho c_{0}^{I I}} \frac{\partial c_{0}^{I}}{\partial \varepsilon} \mu-y\right) \frac{\partial c_{0}^{I}}{\partial \theta}>\left(\frac{1}{\rho c_{0}^{I I}} \frac{\partial c_{0}^{I}}{\partial \theta} \mu-z\right) \frac{\partial c_{0}^{I}}{\partial \varepsilon} \\
& \frac{1}{\rho c_{0}^{I I}} \frac{\partial c_{0}^{I}}{\partial \varepsilon} \mu \frac{\partial c_{0}^{I}}{\partial \theta}-y \frac{\partial c_{0}^{I}}{\partial \theta}>\frac{1}{\rho c_{0}^{I I}} \frac{\partial c_{0}^{I}}{\partial \theta} \mu \frac{\partial c_{0}^{I}}{\partial \varepsilon}-z \frac{\partial c_{0}^{I}}{\partial \varepsilon} \\
& -y \frac{\partial c_{0}^{I}}{\partial \theta}>-z \frac{\partial c_{0}^{I}}{\partial \varepsilon} \\
& y \frac{\partial c_{0}^{I}}{\partial \theta}<z \frac{\partial c_{0}^{I}}{\partial \varepsilon}
\end{aligned}
$$

We have proved in the previous subsection that the above inequality holds true for any $\tau>0$. Thus the slope of $D^{I I}=0$ is larger in absolute value than the slope of $D=0$. 


\section{D.5 Numerator of the $\left.\frac{d \varepsilon}{d \sigma}\right|_{D^{I I}=0}$ Expression}

The numerator of the $\left.\frac{d \varepsilon}{d \sigma}\right|_{D^{I I}=0}$ expression reads:

$$
\begin{aligned}
& \frac{1}{c_{0}^{I I}} \frac{\partial c_{0}^{I I}}{\partial \sigma}+\frac{e^{-\rho \tau}}{\rho} \frac{\partial \tilde{\psi}}{\partial \sigma} \\
& =\frac{\frac{\phi_{x}+(P-\sigma) \phi_{c} \tilde{\psi}}{\phi_{x}+(P-\sigma) \phi_{k}}\left(\phi_{x}+P \phi_{k}\right)+\left(\phi_{x}+P \psi \phi_{c}\right)\left(e^{\rho \tau}-1\right)}{\rho\left[K_{0} e^{\rho \tau}\left(\phi_{x}+P \phi_{k}\right)-\frac{\left(e^{\theta \tau}-e^{-(\rho+\psi) \tau}\right) P \theta \varepsilon e^{\rho \tau}}{(\theta+\psi+\rho)}\right]} \\
& \times \frac{-\rho\left[K_{0} e^{\rho \tau}\left(\phi_{x}+P \phi_{k}\right)-\frac{\left(e^{\theta \tau}-e^{-(\rho+\psi) \tau}\right) P \theta \varepsilon e^{\rho \tau}}{(\theta+\psi+\rho)}\right]\left(\phi_{x}+P \phi_{k}\right)}{\left\{\frac{\phi_{x}+(P-\sigma) \phi_{c} \tilde{\psi}}{\phi_{x}+(P-\sigma) \phi_{k}}\left(\phi_{x}+P \phi_{k}\right)+\left(\phi_{x}+P \psi \phi_{c}\right)\left(e^{\rho \tau}-1\right)\right\}^{2}} \\
& \times \frac{\partial}{\partial \sigma}\left[\frac{\phi_{x}+(P-\sigma) \phi_{c} \tilde{\psi}}{\phi_{x}+(P-\sigma) \phi_{k}}\right]+\frac{e^{-\rho \tau}}{\rho} \frac{\partial \tilde{\psi}}{\partial \sigma} \\
& =-\frac{\left(\phi_{x}+P \phi_{k}\right) \frac{\partial}{\partial \sigma}\left[\frac{\phi_{x}+(P-\sigma) \phi_{c} \tilde{\psi}}{\phi_{x}+(P-\sigma) \phi_{k}}\right]}{\frac{\phi_{x}+(P-\sigma) \phi_{c} \tilde{\psi}}{\phi_{x}+(P-\sigma) \phi_{k}}\left(\phi_{x}+P \phi_{k}\right)+\left(\phi_{x}+P \psi \phi_{c}\right)\left(e^{\rho \tau}-1\right)}+\frac{e^{-\rho \tau}}{\rho} \frac{A \phi_{x} \phi_{k}}{\left[\phi_{x}+(P-\sigma) \phi_{k}\right]^{2}} \\
& =\frac{A \phi_{x} \phi_{k} e^{-\rho \tau}}{\rho\left[\phi_{x}+(P-\sigma) \phi_{k}\right]^{2}} \\
& +\frac{A \phi_{x}^{2}\left(\phi_{x}+P \phi_{k}\right)}{\left[\phi_{x}+(P-\sigma) \phi_{k}\right]^{2}\left\{\frac{\phi_{x}+(P-\sigma) \phi_{c} \tilde{\psi}}{\phi_{x}+(P-\sigma) \phi_{k}}\left(\phi_{x}+P \phi_{k}\right)+\left(\phi_{x}+P \psi \phi_{c}\right)\left(e^{\rho \tau}-1\right)\right\}^{2}} \\
& -\frac{\rho\left(\phi_{x}+P \phi_{k}\right)}{\left\{\frac{\phi_{x}+(P-\sigma) \phi_{c} \tilde{\psi}}{\phi_{x}+(P-\sigma) \phi_{k}}\left(\phi_{x}+P \phi_{k}\right)+\left(\phi_{x}+P \psi \phi_{c}\right)\left(e^{\rho \tau}-1\right)\right\}^{2}}>0 .
\end{aligned}
$$

Hence the numerator is positive.

\section{References}

BASIC (2011) Equitable access to sustainable development: contribution to the body of scientific knowledge. Technical report, BASIC expert group, Bejing, Brasilia, Cape Town and Mumbai

Bretschger L (2015) Energy prices, growth, and the channels in between: theory and evidence. Resour Energy Econ 39:29-52

Bretschger L (2013) Climate policy and equity principles: fair burden sharing in a dynamic world. Environ Dev Econ 18:517-536

Bretschger L, Mollet JC (2015) Prices vs. equity in international climate policy: A broad perspective, CER-ETH Working Paper Series 15/211, ETH Zurich

Bretschger L, Vinogradova A (2014) Growth and Mitigation Policies with Uncertain Climate Damage, CER-ETH Working Paper Series 14/202, ETH Zurich

Bretschger L, Karydas C (2013) Optimum Growth and Carbon Policies with Lags in the Climate System, CER-ETH Working Paper Series 13/184, ETH Zurich

Hicks JR (1932) The Theory of Wages. Macmillan, London

Meinshausen M, Meinshausen N, Hare W, Raper SCB, Frieler K, Knutti R, Frame DJ, Allen MR (2009) Greenhousegas emission targets for limiting global warming to 2 degrees C. Nature 458(7242):115862 
Weitzmann ML (2014) Can negotiating a uniform carbon price help to internalize the global warming externality? J Assoc Environ Resour Econ 1(1/2):29-49

Zhang Z (2009) Multilateral trade measures in a post-2012 climate change regime? what can be taken from the montreal protocol and the WTO?". Energy Policy 37:5105-5112 